\title{
Effects of seismic input, fine crust and existing structure on liquefaction from centrifuge model tests
}

\author{
V. Fioravante ${ }^{1} \cdot$ D. Giretti ${ }^{2}$ (D) S. Airoldi ${ }^{3} \cdot$ J. Moglie $^{3}$
}

Received: 2 April 2020 / Accepted: 28 May 2021 / Published online: 1 July 2021

(C) The Author(s) 2021

\begin{abstract}
The results of dynamic centrifuge tests carried out to study the effects of seismic input, fine crust and existing structure on liquefaction triggering and manifestations are presented. The basic concept of the experimentation was to analyse the seismic behaviour of level ground, saturated, $14 \mathrm{~m}$ deep sandy deposits, homogeneous or stratified, subjected to increasing seismic excitations up to liquefaction, with or without a one degree of freedom structure on shallow foundations. The study was performed in the framework of the European project Horizon2020 “LIQUEFACT".
\end{abstract}

Keywords Centrifuge $\cdot$ Liquefaction $\cdot$ Fine crust $\cdot$ Triggering $\cdot$ Structure

\section{Introduction}

In the framework of the LIQUEFACT project (http://www.liquefact.eu/) a very large series of centrifuge tests were conducted at ISMGEO (Istituto Sperimentale Modelli Geotecnici, Italy). The basic concept of the centrifuge experimentation was to analyze the seismic behavior of level ground, saturated sandy deposits, homogeneous or stratified, subjected to seismic excitations of increasing intensity up to liquefaction and to verify the effectiveness of different liquefaction mitigation techniques. The tests were organized in three series; the first one was focused at investigating the liquefaction triggering conditions and to this aim, three sandy soils, two soil profiles and five different earthquake input motions of increasing intensity, were tested. Some tests were carried out under free field condition, in some other a one degree of freedom structure based on shallow foundations was modelled as well, in order to study the effects of soil-structure interaction. During the second and third test series the effectiveness of vertical and horizontal drains and Induced Partial Saturation in reducing the pore pressure build was analyzed. The final scope of the physical modelling was to produce a consistent set of experimental data on the base of which calibrating and

D. Giretti

daniela.giretti@unibg.it

1 Engineering Department, University of Ferrara, Ferrara, Italy

2 Department of Engineering and Applied Science, University of Bergamo, Bergamo, Italy

3 ISMGEO, Seriate, Italy 
validating numerical tools to be used for parametric analysis (Özcebe et al. 2021; Fasano et al. 2019). The whole experimental campaign is described in Airoldi et al. 2018. All the test results can be found and downloaded at https://www.zenodo.org/record/1281598\#.WmWVOhKjIU.

In this paper the experimental details of centrifuge modelling are presented and some results of the tests carried out to study the liquefaction triggering mechanisms are analyzed. The effect of the following variables on co-seismic and post-seismic behaviour are described:

(i) effect of the input motion,

(ii) effect of a fine crust overlying the sandy deposit and

(iii) effect of presence of a model structure on shallow foundations.

\section{Centrifuge tests overview}

\subsection{Reference prototype, testing soil, model layout.}

One of the focus case study of the LIQUEFACT project is the Emilia region in Italy, where extensive liquefaction phenomena occurred during the 2012 seismic sequence. The two main events of the sequence are the May 20 and May 29 earthquakes, characterized by moment magnitude of $\mathrm{Mw}=6.1$ and $\mathrm{Mw}=5.9$, respectively (www.iside.rm.ingv.it). The most relevant liquefaction manifestations were observed during the May 20 shake in the Ferrara Province, at sites located about $15 \mathrm{~km}$ SE of the epicenter. Liquefaction evidences consisted in craters, sand boils, surface cracks and lateral spreading. The soils which experienced liquefaction are shallow (within 12-15 $\mathrm{m}$ from the ground surface) river deposits of sandy silt, silty sand and sand, topped by a clayey silt layer of lower permeability, up to $2 \mathrm{~m}$ thick. The ground water table is close to the soil surface (Calabrese et al. 2012; Giretti and Fioravante 2017).

The ground conditions at those sites were taken as a reference for the centrifuge models. It was established to test level ground sandy deposits, about $14 \mathrm{~m}$ deep, either homogeneous or with a top cap of fine grained soil, $1.5 \mathrm{~m}$ thick. The ground water table was set at the soil surface. To reproduce the reference prototype in the centrifuge, a geometrical scaling factor $\mathrm{N}=50$ was adopted and the models were subjected to a centrifugal acceleration of $50 \mathrm{~g}$, imposed in correspondence of the base of the models.

The test here presented were carried out using a well-known Italian clean sand (Ticino Sand, herein referred to as TS) extensively used in the last 40 years for geotechnical experimentations.

TS is a uniform coarse to medium sand (Fig. 1) made of angular to sub-rounded particles and composed of $30 \%$ quartz, $65 \%$ feldspar and 5\% mica. The angle of sharing resistance at critical state is $34^{\circ}$ (stress ratio at critical state $\mathrm{M}=1.36$ ), the critical state parameters are $\Gamma=0.923, \lambda=0.046 \alpha=0.5$ (according to the equation of the critical state line in e-p' plane by Li and Wang 1998). A detailed description of its static and dynamic properties can be found in Fioravante and Giretti (2016). Figure 1 shows the grain size distribution of TS, whose main index properties and hydraulic conductivity to water at the test void ratio are listed in Table 1. 


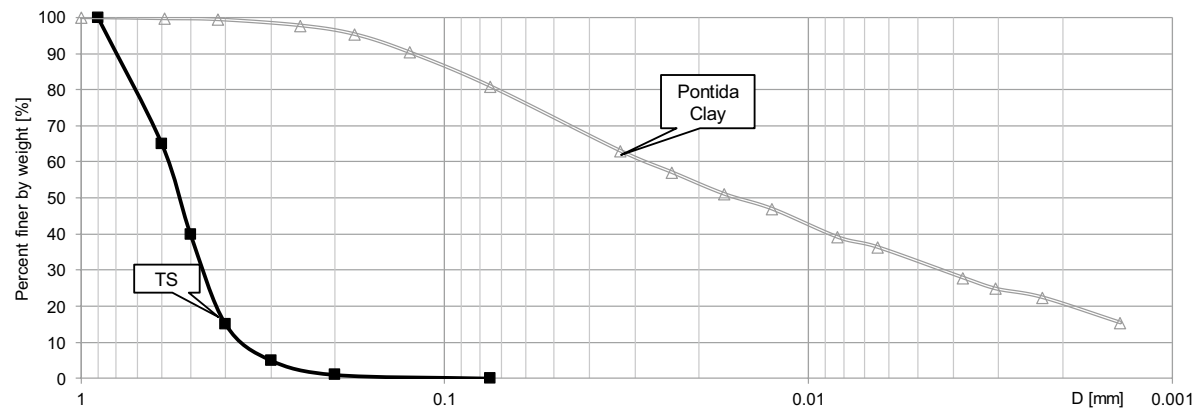

Fig. 1 Grain size distribution of Ticino Sand TS and Pontida Clay

Table 1 Characteristics of the test soils

\begin{tabular}{llllllllll}
\hline Sand & $\gamma_{\min }\left(\mathrm{kN} / \mathrm{m}^{3}\right)^{*}$ & $\gamma_{\max }\left(\mathrm{kN} / \mathrm{m}^{3}\right)^{* *}$ & $\mathrm{e}_{\min }$ & $\mathrm{e}_{\max }$ & $\mathrm{G}_{\mathrm{s}}$ & $\mathrm{D}_{50}(\mathrm{~mm})$ & $\mathrm{FC}(\%)$ & $\mathrm{IP}(\%)$ & $\mathrm{K}(\mathrm{m} / \mathrm{s})^{* * *}$ \\
\hline Ticino (TS) & 13.64 & 16.67 & 0.574 & 0.923 & 2.68 & 0.53 & 0 & - & $2 * 10^{-3}$ \\
Pontida Clay & - & - & - & - & 2.77 & 0.017 & 81 & 11 & $10^{-9}-10^{-10}$ \\
\hline
\end{tabular}

*ASTM 4254, **ASTM 4253, *** value at the test void ratio reported in Table 2

Figure 2 shows the model schemes of the tests discussed herein. The sandy deposit modeled was arranged in two different ground conditions: Model 1 (M1, Figs. 2a, b) represented a homogeneous sand profile; in Model 2 (M2, Fig. 2c) the same sand deposit was topped by a $1.5 \mathrm{~m}$ thick fine grained layer of lower permeability (all measures in Fig. 2 are at prototype scale). In both cases the groundwater table coincided with the ground surface.

The fine grain layer overtopping the sand in M2 model was reconstituted using Pontida Clay (Fioravante and Jamiolkowski 2005, grain size curve in Fig. 1), which is a low plasticity kaolinitic clayey silt, whose main characteristics are: specific density, $\mathrm{G}_{\mathrm{s}}=2.77$, liquid limit, $\mathrm{w}_{\mathrm{L}}=24 \%$, plastic limit, $\mathrm{w}_{\mathrm{P}}=13 \%$. Grain size analyses indicate a prevalence of siltsize particles ( $59 \%$ by weight) with $22 \%$ clay size particles and $19 \%$ sand. Pontida clay is composed by 25-30\% illite, 20-25\% kaolinite, 20-25\% quartz, $12 \%$ calcite and dolomite, less than $10 \%$ feldspar. The angle of shearing resistance at critical state is $33^{\circ}(\mathrm{M}=1.33)$, the critical state parameters are $\Gamma=0.7, \lambda=0.04$ (according to a logarithmic equation of the critical state line in the e-p' pane). The hydraulic conductivity to water of Pontida Clay in the test condition is in the range of $10^{-9}-10^{-10} \mathrm{~m} / \mathrm{s}$ (Table 1 ).

In some tests a simple structure on shallow foundations (F) was modelled, as sketched in Fig. 2d. The structure was a single degree of freedom system composed by an 'oscillating system' founded on two beams rigidly connected by rigid bars (not in contact with the soil). Each foundation beam, made of aluminium, was $1.15 \mathrm{~m}$ wide and $11.5 \mathrm{~m} \mathrm{long}$, at the prototype scale, while the total width of the foundation system was $7.15 \mathrm{~m}$. The superstructure was $4 \mathrm{~m}$ high, $6 \mathrm{~m}$ wide and $8 \mathrm{~m}$ long. The lumped mass was supported by two side walls made of steel. The foundations were embedded $1.5 \mathrm{~m}$ from the ground surface. The mass, geometry and flexural stiffness of the oscillating system, were designed to simulate the frequency of a two-storey masonry building. The total mass and natural frequency of the prototype structure are 250 tons and $3.1 \mathrm{~Hz}$, respectively (in prototype scale), whereas 


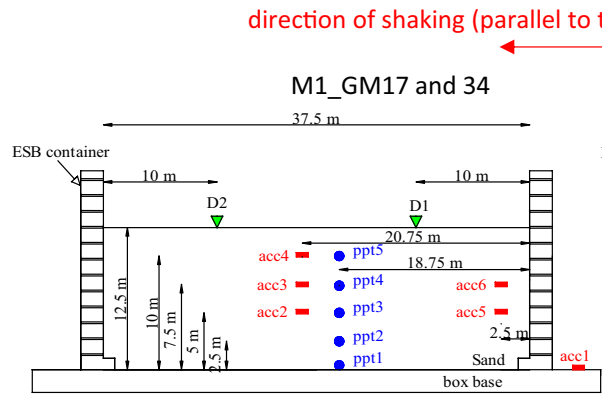

(a)

M2_GM31

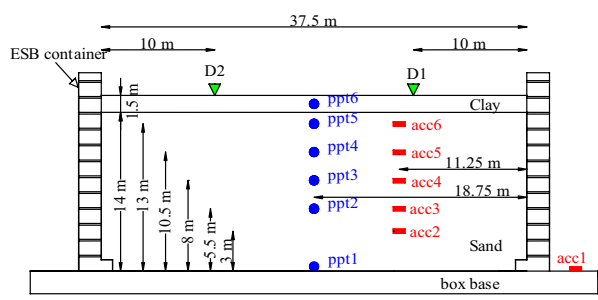

(c)

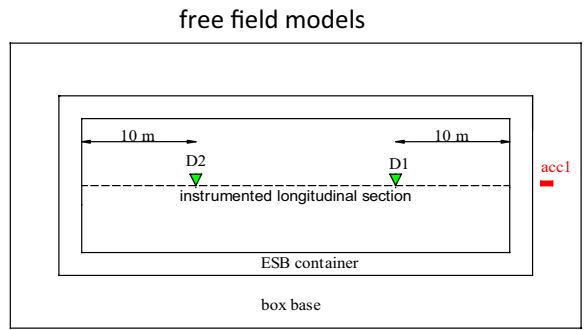

(e)

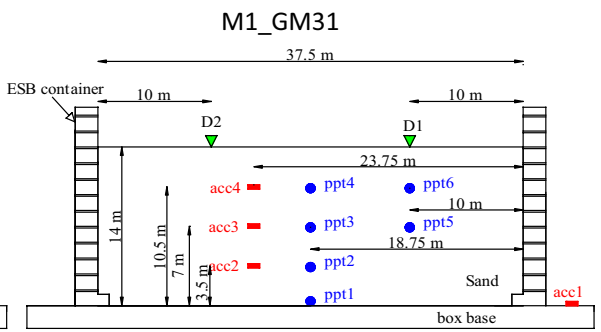

(b)

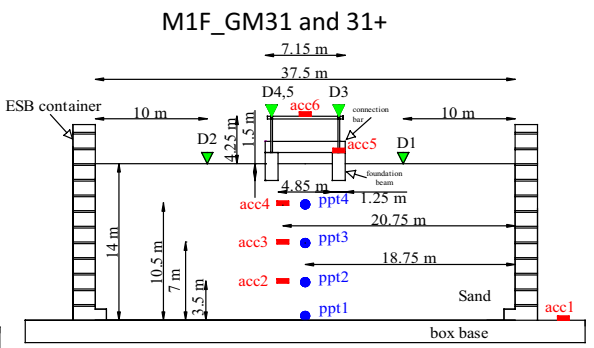

(d)

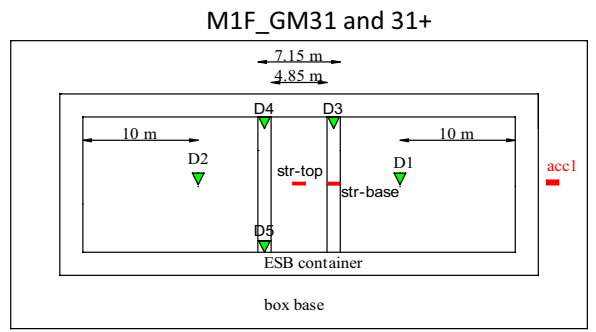

(f)

Fig. 2 Frontal sections and top views of models after sand deposition. Frontal sections: a M1_GM17 and 34, b M1_GM31, c M2_GM31, d M1F_GM31 and 31+. Top views: $\mathbf{g}$ all free field models, h models with the structure. Prototype units

Table 2 Test program

\begin{tabular}{llllll}
\hline Test number* & Model type & Density (\%) & Void ratio e(-) & Input signal & ID \\
\hline 1 & M1 & 47 & 0.761 & GM17 & M1_GM17 \\
2 & & 50 & 0.748 & GM34 & M1_GM34 \\
3 & 47.5 & 0.757 & GM31 & M1_GM31 \\
11 & & 50.5 & 0.747 & GM31 & M2_GM31 \\
13 & M2 & 49 & 0.752 & GM31 & M1F_GM31 \\
14 & M1F with structure & 53 & 0.738 & GM31+ & M1F_GM31+ \\
\hline
\end{tabular}

*Test number according to the original test sequence of the LIQUEFACT project 
the natural frequency of the sandy deposit is around $2.3 \mathrm{~Hz}$. Table 2 lists the main characteristics of the test described in this paper.

\subsection{The ISMGEO seismic geotechnical centrifuge and model container}

The ISMGEO geotechnical centrifuge is a beam centrifuge made up of a symmetrical rotating arm with a diameter of $6 \mathrm{~m}$, a height of $2 \mathrm{~m}$, a width of $1 \mathrm{~m}$, and a nominal radius of about $2.2 \mathrm{~m}$ to the model base. An outer fairing covers the arm and they concurrently rotate to reduce air resistance and perturbation during flight. The centrifuge has a $240 \mathrm{~g}$-ton capacity, i.e. the machine has the potential of reaching an acceleration of $600 \mathrm{~g}$ holding a payload of $400 \mathrm{~kg}$ (Baldi et al. 1988).

At each side, the symmetric arm holds a swinging platform which carries the model container, for static test at one side, for dynamic test at the other. At one side of the arm is fixed a single degree of freedom shaking table, whose reaction base is the rotating arm itself. The swinging platform which holds the model for dynamic tests is moved in contact with the table in flight and released before starting the dynamic excitation. The shaking table can work under an artificial acceleration field up to $100 \mathrm{~g}$ and can provide excitations at frequencies up to $500 \mathrm{~Hz}$ and seismic accelerations up to $50 \mathrm{~g}$. The shaker can reproduce real strong motions at the model scale (Airoldi et al. 2016).

An Equivalent Shear Beam (ESB) box (Zeng and Schofield 1996; Brennan et al. 2006) was specifically designed and constructed for the tests of LIQUEFACT project. The box is composed by twelve aluminum rectangular frames with a height of $25 \mathrm{~mm}$ each, separated by eleven $3.36 \mathrm{~mm}$ thick rubber inter-layers. This configuration returns a total container height of $337 \mathrm{~mm}$. It's worth noting that, in flight, the long side of the container is vertical and parallel to the rotation axis of the centrifuge, so that the distortion effect due to the rotation does not affect the central section of the model along which the instruments were located. In addition, the shaking direction is parallel to the rotation axis of the centrifuge, thus problems related to Corioli's acceleration are avoided.

\subsection{Model reconstitution, saturation and instrumentation}

The soil models were reconstituted at low density by pluviating in air the dry sand into the ESB container at a height of fall of about $3 \mathrm{~cm}$. The height of fall was calibrated in order to obtain a relative density at $1 \mathrm{~g}$ of about $40 \%$. Weight and volumes of sand were constantly measured during the reconstitution. The 1-g density was lower than the model density before shaking, since during the subsequent phases (saturation, increasing g-level during the centrifuge spin-up) the soil density increased. The position of the top surface was measured at the end of reconstitution and during the subsequent test steps using displacement transducers to calculate and update the average value of relative density during all the experimental phases. The values of density in Table 2 refer to the pre-shaking condition.

Saturation of the models was carried out at the end of the sand deposition using a viscous fluid. The use of a viscous fluid rather than water was necessary due to avoid the discordance between the scaling ratios for time in dynamic phenomena and in diffusion phenomena (Kutter 1995) and to model properly both the dynamic stage and the earthquake induced reconsolidation phenomena. The tested physical models were geometrically scaled down of a factor $\mathrm{N}=50$, in consequence it was necessary to adopt a porous fluid with a viscosity 50 times the water viscosity. A solution of water and 
hydroxypropyl methylcellulose (HPMC) with a concentration of $2 \%$ was adopted. This concentration gives a kinematic viscosity of $50 \mathrm{cSt}$ (water kinematic viscosity $\approx 1 \mathrm{cSt}$ at $20^{\circ}$ ) and a unit weight of $9.84 \mathrm{kN} / \mathrm{m}^{3}$, that is approximately the unit weight of water. The correct concentration value was verified by viscometer tests. With pore fluid viscosity $\mathrm{N}$ times the water viscosity, permeability may be treated as the same in the model and in the prototype.

The saturation was carried out as follows (see the sketch in Fig. 3): the ESB box with the dry sand layer was placed in the centrifuge basket and covered with a steel plate, sealed and connected to a vacuum pump. The reservoir with the fluid was installed above the ESB box and connected by two pipes, one from the reservoir bottom to the ESB bottom for the fluid flow, one pipe from the ESB top to the reservoir top to keep the two containers under the same level of vacuum (about $-60 \mathrm{kPa}$ ). The adopted configuration produced an upward fluid flow through the model of low gradient, whose rate was kept constant until the permeated volume of fluid was at least equal to the estimated soil volume of voids. This stage lasted at least $7 \mathrm{~h}$. During the whole saturation stage the internal membrane of the box was sealed against the walls by means of about $-80 \mathrm{kPa}$ of vacuum. At the end of the saturation, the ground surface settlements were carefully measured.

In type M2 models, the top layer of Pontida Clay was placed above the sand surface at the end of the sand saturation. To reconstitute the fine grained top layer, dry Pontida Clay powder and deaired water were mixed to form a slurry with a water content equal to $42 \%$ (1.75 times the liquid limit). Mixing was continued for about two hours under a vacuum of $750 \mathrm{~mm} \mathrm{Hg}$. The clay slurry was then transferred into the consolidometer. The height of specimen after consolidation was approximately equal to $30 \mathrm{~mm}$. After the consolidation the specimen was unloaded, removed from the consolidometer and placed above the sand model surface just before the test. During this phase, is possible that the clay did lost its water content along the contours giving rise to suction tensions, which in turn may have locally desaturated the sand surface. The layer was not
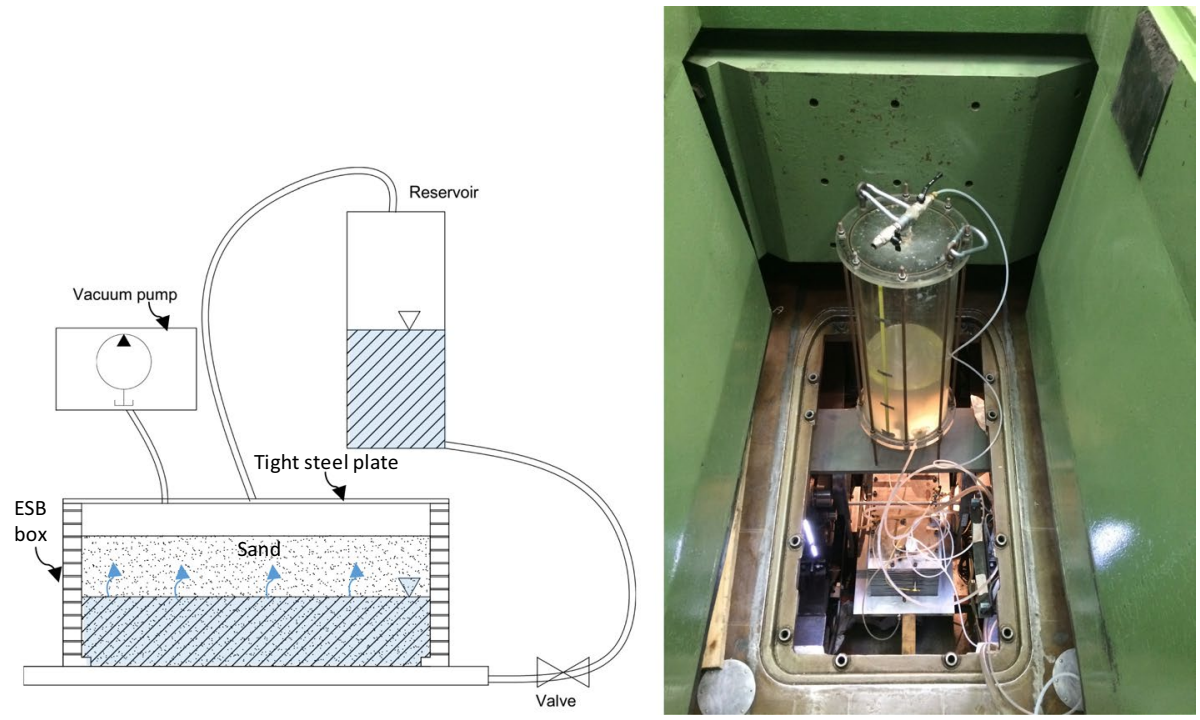

Fig. 3 Scheme and picture of the saturation system (saturation carried out at $1 \mathrm{~g}$, with the model in horizontal position) 
instrumented with tensiometers. Under the centrifugal field the clay layer had an over consolidation ratio OCR larger than 20 .

As shown in Fig. 2, the tested models were equipped with miniaturized accelerometers (acc), pore pressure transducers (ppt) and displacement transducers (D) to measure horizontal accelerations along the shaking direction, fluid pressure and settlement, respectively.

In particular, both PCB 352C22 piezoelectric ceramic uniaxial accelerometers (single axis, measurement range $\pm 5000 \mathrm{~g}$, sensitivity $10 \mathrm{mV} / \mathrm{g}$ ) and ADXL78 MEMS by Analog Devices (single axis, measurement range $\pm 70 \mathrm{~g}$, sensitivity $25.65 \mathrm{mV} / \mathrm{g}$ ) were used. As miniaturized ppts, the EPB-PW by TE Connectivity sensors, equipped with a sintered bronze filter, were employed (pressure range 0-15 bar, sensitivity $12.5 \mathrm{mV} / \mathrm{V}$ ). The linear displacement transducers adopted are potentiometers with range $0-50 \mathrm{~mm}$ and sensitivity $0.01 \mathrm{mV} / \mathrm{mm}$.

The transducers were installed in the models during the deposition stage, following each model design specifications. Sand pouring was stopped at the level at which the sensors should be installed, the ground surface was levelled and the correct position within the container was measured. The sensors were installed along the longitudinal central axis of the container, in order to minimize the boundary effects on the measurements. In this way, all measurements can be referred to the same section under plane strain conditions. The position and configuration of sensors was changed from test to test depending on the specific test characteristics. In general, at least a vertical array of unidirectional accelerometers was installed inside the models to measure seismic wave propagation from bottom to top. The sensitive direction was parallel to the shaking direction of the table. A further accelerometer was fixed to the base of the model container in order to measure the time history applied by the shaking table. A vertical array of miniaturized pore pressure transducers was also installed in the models and allowed the pore pressure evolution during and after the shocks to be monitored. Two linear displacement transducers measured the soil surface vertical displacements (in test interpretation their measures have been averaged). The transducers tip rested above a thin and light plate, necessary to minimize the tip sinking. When present, also the structure was instrumented with two accelerometers and three displacement transducers (displacements measures averaged). The data acquisition chain was completed by a National Instruments DAQ system and a Personal Computer installed in the centrifuge and connected to the control room by a wireless system. During the application of seismic shocks all data were recorded with a sampling rate of $5 \mathrm{kHz}$.

\subsection{Input signals}

A specific site response analysis was carried out (Chiaradonna et al. 2018) in order to provide a series of ground motions, corresponding to different seismic hazard levels (return period $\mathrm{T}_{\mathrm{r}}=475,975$ and 2475 years), to be applied to the centrifuge models via the shaking table. The motions were computed referring to the deep seismic profile of the reference prototype area in the Emilia Romagna region. Calculations were performed and verified using independent approaches. The acceleration time histories were computed at the depth of $15 \mathrm{~m}$, i.e. at the base of the sandy deposit that was modelled in centrifuge.

Among the 21 signals analyzed, a limited number of ground motions (GMs) of increasing intensity were selected for the centrifuge tests, as more suitable to the shaking table capabilities.

To be used in centrifuge seismic tests, the computed signals required specific adaptation to shaking table technical specifications. The maximum frequency and acceleration 


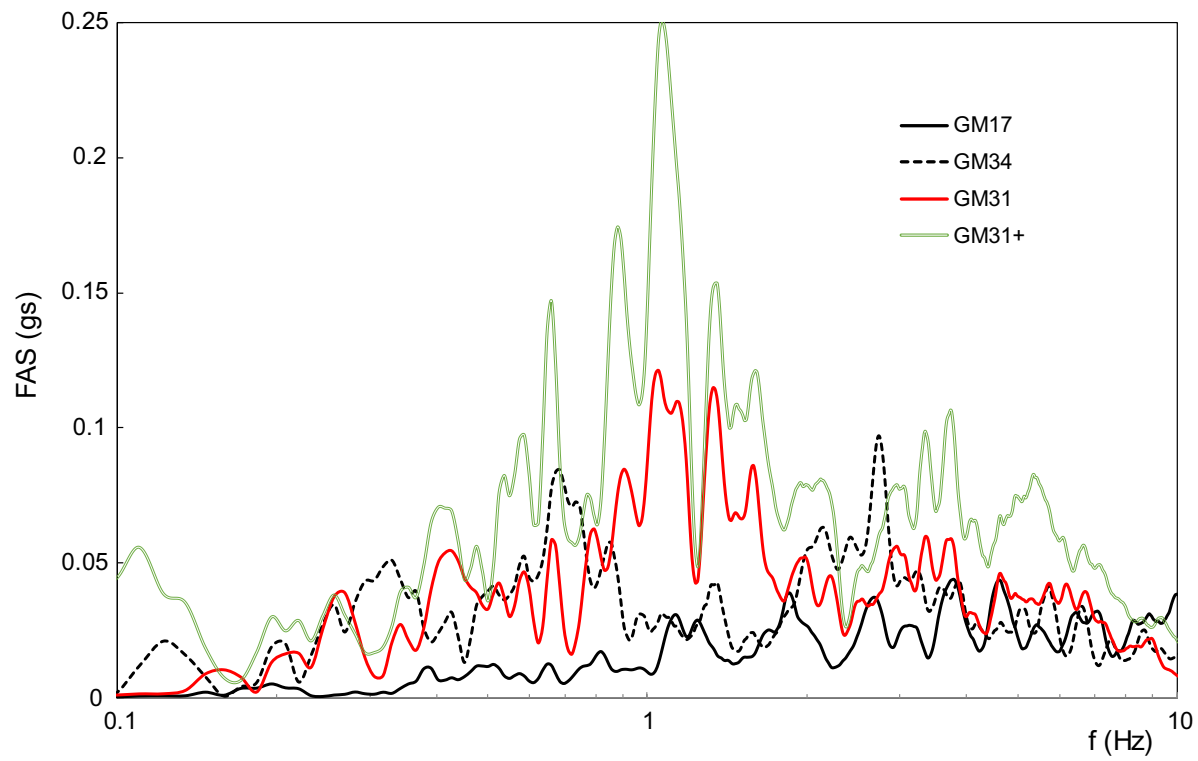

Fig. 4 FAS of the motions reproduced by the shaking table. Prototype units

Table 3 Input motion characteristics

\begin{tabular}{llll}
\hline Test ID & PGA $(\mathrm{g})$ & $\mathrm{d}_{90}(\mathrm{~s})$ & $\mathrm{I}_{\mathrm{A}, \max }(\mathrm{m} / \mathrm{s})$ \\
\hline M1_GM17 & 0.215 & 15.09 & 0.348 \\
M1_GM34 & 0.222 & 24.23 & 0.451 \\
M1_GM31 & 0.198 & 18.63 & 0.601 \\
M2_GM31 & 0.243 & 21.76 & 0.673 \\
M1F_GM31 & 0.216 & 17.24 & 0.481 \\
M1F_GM31 & 0.292 & 22.48 & 1.844 \\
\hline
\end{tabular}

GMID $=$ Ground Motion ID; PGA = peak ground acceleration; $\mathrm{d}_{90}=$ duration calculated on the base of Arias Intensity; $\mathrm{I}_{\mathrm{A}, \max }=$ maximum Arias Intensity

values in flight were limited to $500 \mathrm{~Hz}$ and $15 \mathrm{~g}$ respectively. Those values correspond to $10 \mathrm{~Hz}$ and $0.3 \mathrm{~g}$ at prototype scale. The computed time histories had all maximum acceleration values lower than $0.3 \mathrm{~g}$. On the other hand, the records contained a certain amount of information for $\mathrm{f}>10 \mathrm{~Hz}$; thus a low-pass filter was used to reduce the spectral information for higher frequencies.

The selected GMs, properly scaled, were applied to the models to investigate the liquefaction triggering conditions Figure 4 reports the examples of the Fourier Amplitude Spectra (FAS) of the motions carried out by the shaking table during the tests described in this paper (GM17, 34, 31, 31+). GM31+ is an amplified version of GM31. As evidenced in the Figure, the energy content of GM17 is concentrated at frequency higher than $1 \mathrm{~Hz}$, while GM34 has discrete energy also at lower frequency. The energy released 
by GM31 and GM31 + is mainly concentrated between 0.8 and $2 \mathrm{~Hz}$. Table 3 lists the main properties of the input motions applied to models herein discussed.

\section{Test results description and interpretation}

All the records discussed in this section are plotted in prototype units.

The effect of the following variables on co-seismic and post-seismic behaviour are described:

(1) input motion.

(2) fine crust at the surface.

(3) presence of a structure on shallow foundation.

\subsection{Homogeneous models M1 under different ground motions}

In this section is compared the behaviour of homogeneous models (M1) subjected to different ground motions: GMs 17, 34 and 31, model scheme in Fig. 2a, b. Table 3, reports the main characteristics of the three earthquakes run by the shaking table in terms of maximum acceleration, duration and max Arias intensity $\mathrm{I}_{\mathrm{A}, \max }$. GM17, 34 and 31 had similar PGA but increasing $\mathrm{I}_{\mathrm{A}, \max }$.

The pore pressure ratios Ru measured in the central axis of the three models and the average superficial settlement $S_{\text {avg }}$ are plotted as a function of time in Figs. 5, 6 and 7, where $\mathrm{Ru}$ is computed as a function of the excess pore pressure $\Delta \mathrm{u}$ measured by ppts:
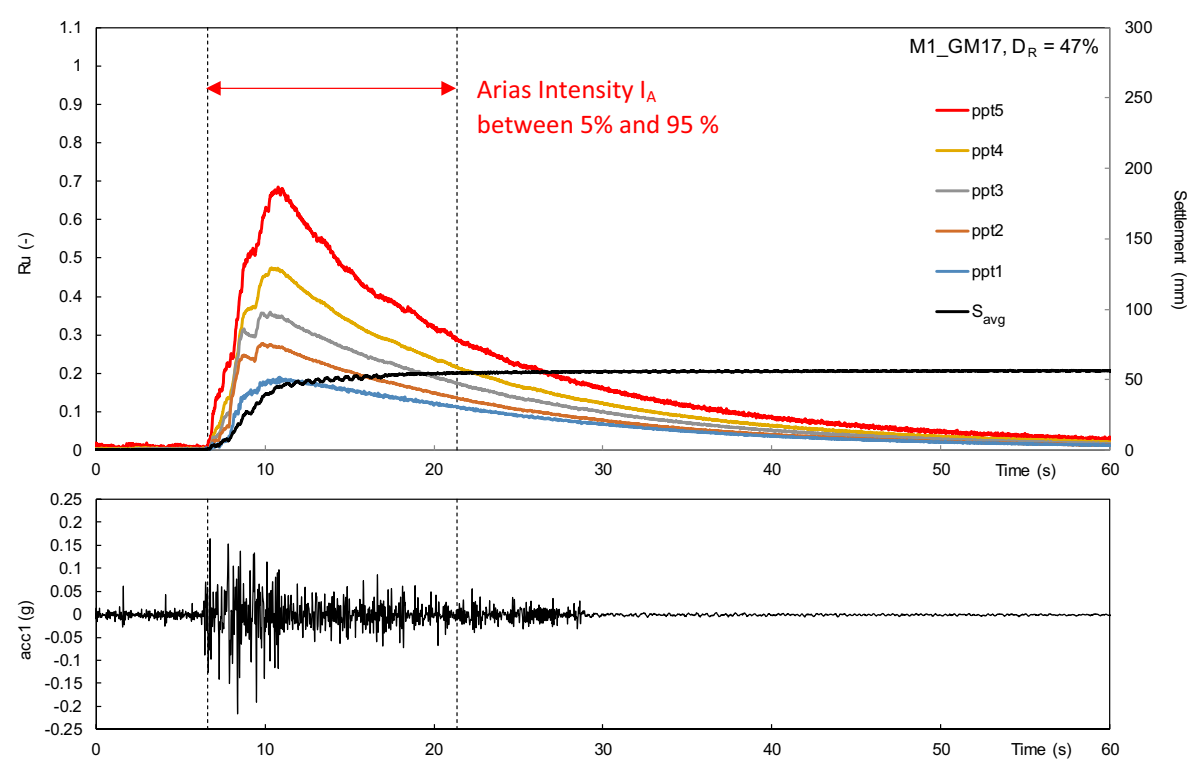

Fig. 5 Pore pressure ratio $\mathrm{Ru}$, average settlement $\mathrm{S}_{\text {avg }}$ and input time history of model M1_GM17. Prototype scale 

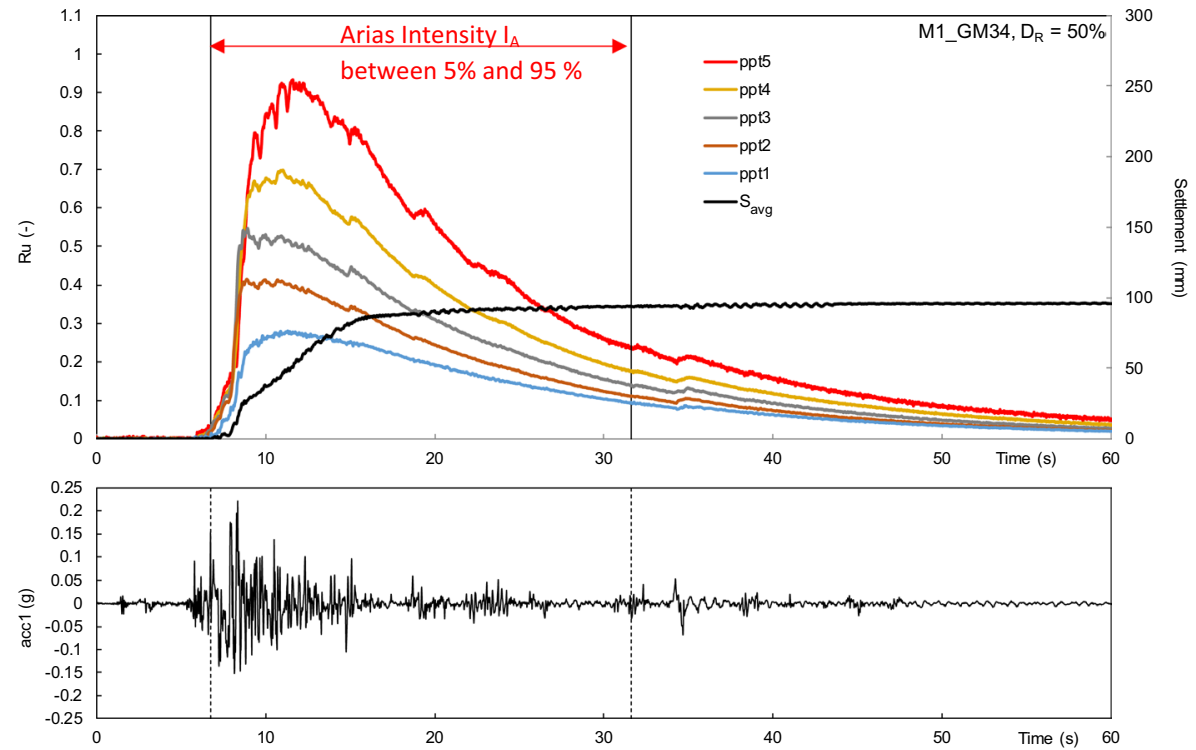

Fig. 6 Pore pressure ratio Ru, average settlement $S_{\text {avg }}$ and input time history of model M1_GM34. Prototype scale
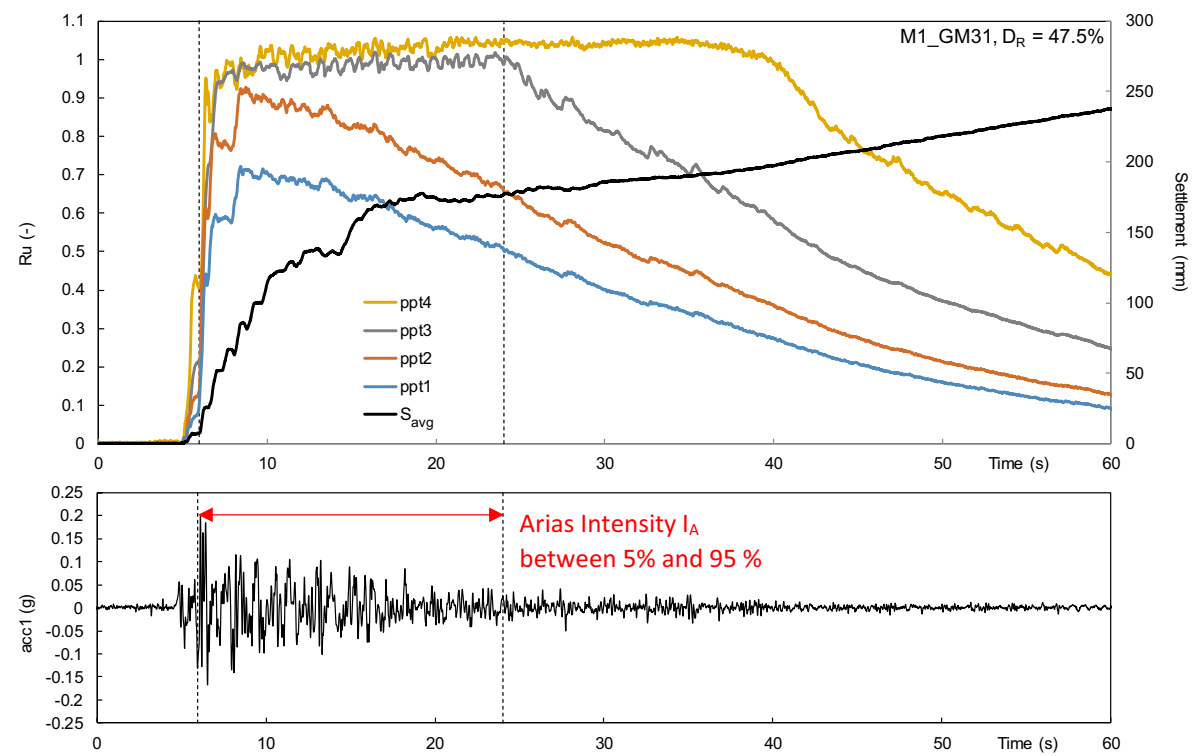

Fig. 7 Pore pressure ratio Ru, average settlement $S_{\text {avg }}$ and input time history of model M1_GM31. Prototype scale 


$$
R u=\frac{\Delta u}{\sigma_{v 0}^{\prime}}
$$

$\sigma_{\mathrm{v} 0}^{\prime}$ being the vertical effective stress acting at the depth of each sensor before ground shaking. In the computation of $\sigma_{\mathrm{v} 0}^{\prime}$ it was accounted for: (i) the estimated current embedment depth of the instruments and (ii) the sand average unit weight, both achieved at the end of the in-flight consolidation.

Figures 5, 6 and 7 also show the input time history of acceleration measured by acc1. The dotted lines on the charts indicate the beginning and end of the base motion, i.e. the time instants at which $5 \%$ and $95 \%$ of the $\mathrm{I}_{\mathrm{A}}$ was released, respectively.

Figure 8 shows the amplification functions (or spectral ratios SRs) of the three models under discussion, computed as the ratio between the FAS of the accelerations recorded by the most superficial accelerometer and the base accelerometer (acc1).

In test interpretation, the liquefaction criterion has been assumed as the point when $\mathrm{Ru}$ approaches 1 . However, the value of $\mathrm{Ru}$ is influenced by the computed value of vertical effective stress at the depth of ppts, whose position is exactly known at $1 \mathrm{~g}$ at the end of model reconstitution, but in flight is influenced by the vertical deformation that the model experiences after reconstitution. The pre-shock depth of sensors was deduced by the superficial settlement previously experienced by the model and it was used to compute the relating vertical effective stress. As a consequence, Ru larger than 0.9 and constant for a period of time, was considered as indicator of soil liquefaction and a minor error were accepted.

According with this criterion, full liquefaction was achieved only by model M1_GM31 at mid depth (ppt3) and near the ground surface (ppt4), as highlighted in Fig. 7.

In this model, at all depths the $\Delta \mathrm{u}$ increased up to a maximum value in the first $2.5 \mathrm{~s}$. Then, at ppts 1 and 2 the excess pore pressure started to decay, almost simultaneously and well in advance respect to the end of the ground motion, while ppts 3 and 4, remained at

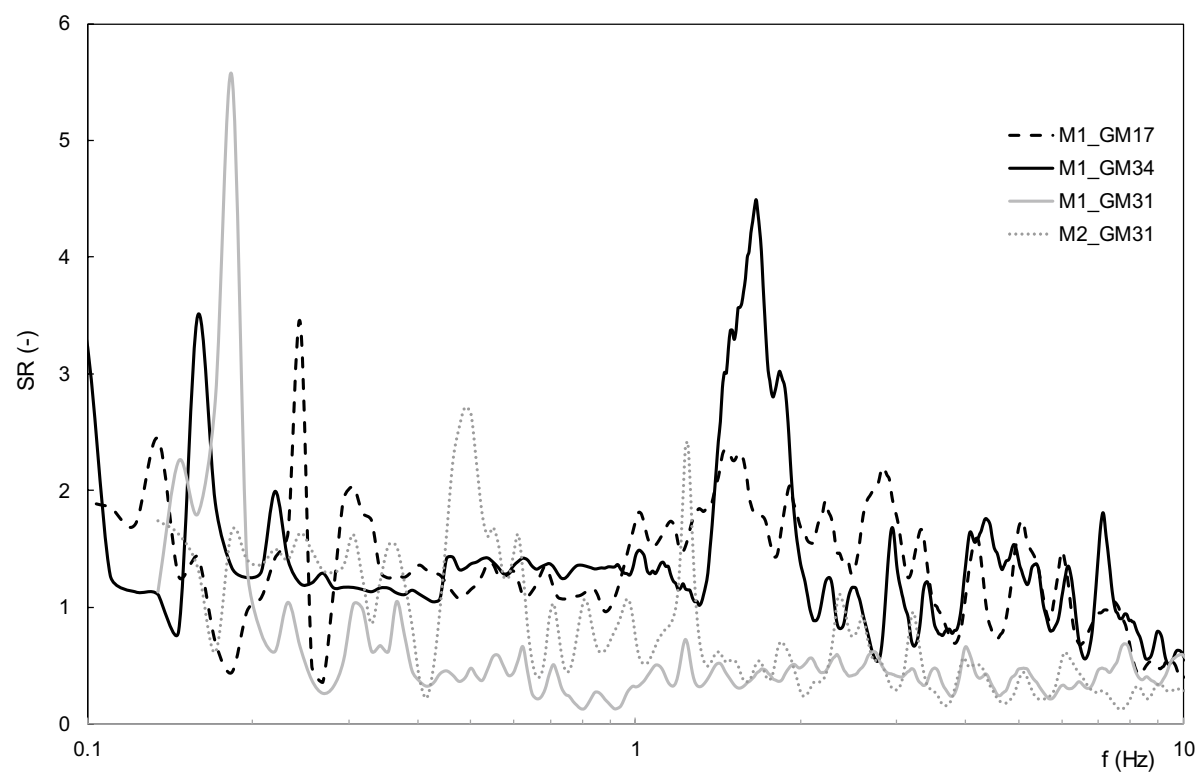

Fig. 8 Models M1_GM17, M1_GM34, M1_GM31 and M2_GM31: spectral ratios SR computed dividing the FAS of the superficial accelerometer by the FAS of the base accelerometer. Prototype scale 
the maximum $\Delta \mathrm{u}$ all along the dynamic excitation and afterwards. Liquefaction triggered first near the ground surface and then quickly propagated downward but didn't reach ppt2, so that, after $2.5 \mathrm{~s}$, the model resulted split in two halves: the upper part fluidified, the bottom part at the solid state (Scott 1986). This condition lasted up to the end of the ground motion, immediately after which ppt3 started to measure a $\Delta \mathrm{u}$ decrease, indicating that solidification had occurred at that depth. The solidification front reached ppt 4 in $16 \mathrm{~s}$. If the solidification velocity is computed as a function of the time difference between the triggering of $\Delta \mathrm{u}$ decay at the depths of ppts 3 and 4 , and this velocity is assumed constant up to the soil surface, it may be derived that after the end of ground shaking the solidification front moved upwards at $0.2 \mathrm{~m} / \mathrm{s}$ and reached the soil surface in $31 \mathrm{~s}$. After that, all the soil column was solidified and involved in a reconsolidation process to dissipate the excess pore pressure.

The starting of $\Delta \mathrm{u}$ reduction from the bottom of the model upward after only few seconds of seismic excitation, indicates that the response of the sand to the dynamic excitation is a partially-drained phenomenon and that as the soil is dynamically strained and starts to generate excess pore pressure, at the same time it starts to dissipate (Liu and Dobry 1997; Adamidis and Madabhushi 2018). During the earthquake, the measured $\Delta \mathrm{u}$ is a balance between generation and dissipation. At ppts 1 and 2, after $2.5 \mathrm{~s}$ of shaking, dissipation prevailed on generation, triggering an upward fluid flow, i.e. from higher excess pore pressure towards lower values at the surface. In the upper part of the model (ppts 3 and 4), the generation induced by the shaking and the inflow from greater depths, prevailed on dissipation, the hydraulic gradient reached the critical value and the soil liquefied, and lingered in a fluidified state for the duration of the ground motion (ppt3) or longer (ppt4).

This mechanism is highlighted also by the development of the surface settlement, which at the end of recording was $S_{t}=265 \mathrm{~mm}: 30 \%$ of $S_{t}$ developed during the first $2.5 \mathrm{~s}$, when all the ppts registered an increasing of $\Delta \mathrm{u} ; 67 \%$ of $\mathrm{S}_{\mathrm{t}}$ developed during the ground motion as a combined effect of drainage, sedimentation and reconsolidation mechanisms; only $33 \%$ of $\mathrm{S}_{\mathrm{t}}$ was post-seismic settlement due to solidification and reconsolidation processes.

Soil liquefaction caused an attenuation of the applied input motion from mid depth upward, as demonstrated by the stress ratio SR of the shallower accelerometer (acc4, straight gray line) shown in Fig. 8, which assumes values lower than 1 at all frequency, indicating a significant loss of stiffness of the sand at that depth.

As to models M1_GM17 and M1_GM34, the applied ground motions had very similar PGA to GM31 but lower $\mathrm{I}_{\mathrm{A}, \max }$ (Table 3). In particular $\mathrm{I}_{\mathrm{A}, \max }$ is 0.348 for GM17, 0.451 for GM34 and 0.601 for GM31. Model M1_GM17 developed a max Ru of 0.7 at the depth pf ppt5, the max Ru of model M1_GM34 was 0.9 at the same ppt. In both models, all ppts reached a peak $\mathrm{Ru}$ almost simultaneously, in about $5 \mathrm{~s}$, then $\Delta \mathrm{u}$ started to decrease. Generation of excess pore pressure was the dominant mechanism only in the first few seconds, then dissipation and drainage prevailed and at all depths the upward outcoming fluid flow overcame the incoming flow, and the model didn't experienced liquefaction, as confirmed also by the amplification functions of the shallower accelerometer in Fig. 8. GM17 (dotted black line) was amplified at all frequency as the shock propagated upward, even if spectral ratios SR not larger than 2.5 can be observed in the frequency range 1-3 Hz. The higher $\mathrm{Ru}$ values reached by GM34 (straight black line) are reflected by modest amplification at all frequency except those in the range $1.4-1.8 \mathrm{~Hz}$. Overall, high values of $\Delta \mathrm{u}$, even if not as high as to induce liquefaction, softened remarkably the soil behavior, changing significantly the frequency of the deposit. 


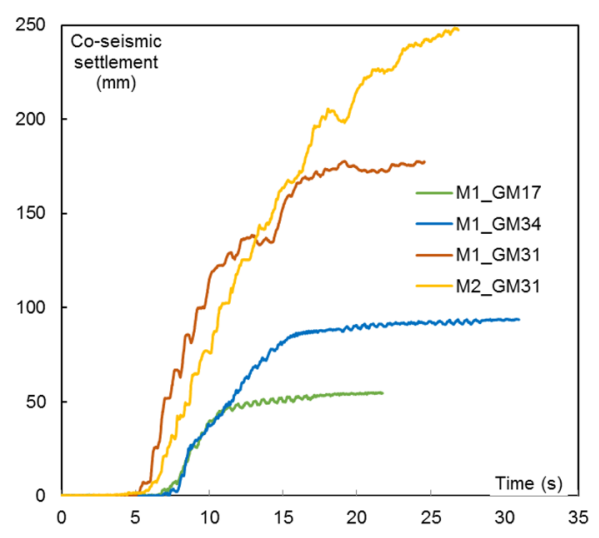

(a)

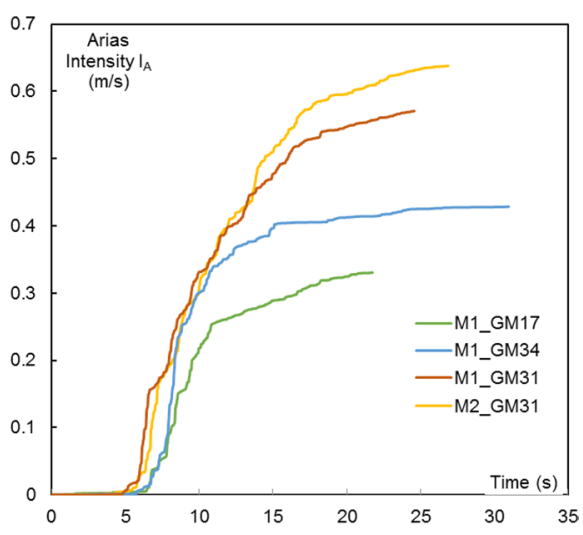

(b)

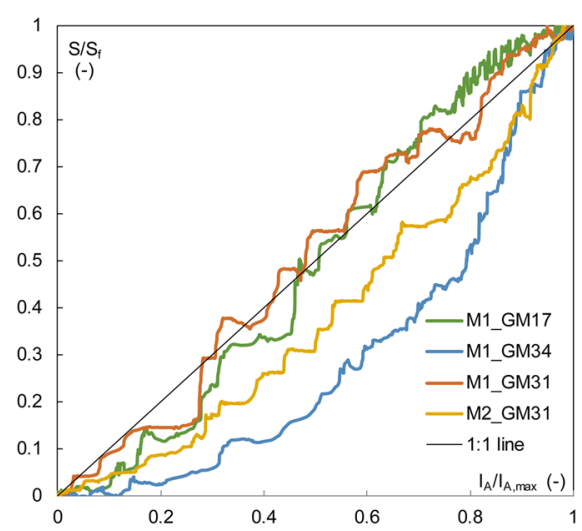

(c)

Fig. 9 Models M1_GM17, M1_GM34, M1_GM31, M2_GM31: a co-seismic settlement b Arias Intensity time history and $\mathbf{c}$ normalized settlement vs normalized Arias Intensity. Prototype scale

The superficial settlements were in both models almost entirely co-seismic. In particular, a significant share of the final settlement $\mathrm{S}_{\mathrm{t}}$ developed during the initial excess pore pressure accumulation phase: $84 \%$ in model M1_GM17, 50\% in model M1_GM34.

In general, the settlement due to rapid drainage during the initial earthquake stage of high hydraulic gradient development, resulted to be greater than post-earthquake reconsolidation settlement. When liquefaction occurred, as in M1_GM31 model, also the sedimentation settlement represented a significant share of $\mathrm{S}_{\mathrm{t}}$.

From a comparison between the co-seismic settlement and the Arias Intensity $\mathrm{I}_{\mathrm{A}}$ released by the input earthquakes, it can be observed a good correlation, as shown in Fig. 9. In Fig. 9a is shown the average surface settlement measured during the seismic excitation; in Fig. 9b the time histories of $\mathrm{I}_{\mathrm{A}}$; in Fig. 9c the co-seismic settlement normalized over its end-of-earthquake value $S_{f}$ is plotted versus the normalized $I_{A} / I_{A \text {,max }}$. Arias intensity and co-seismic settlement time histories have very similar shape and a 1:1 linear relationship exists between the two normalized variables, with a $\mathrm{R}^{2}$ larger than 0.9 .

Previous studies have highlighted the existence of a correlation between liquefaction induced building settlement and an intensity measures linked to Arias intensity, or between 
$\mathrm{Ru}$ and $\mathrm{I}_{\mathrm{A} \text {,max }}$, suggesting the possibility of using alternative earthquake parameter than PGA to evaluate the consequence of liquefaction (Kayen and Mitchell 1997; Green and Mitchell 2003; Kramer and Mitchell 2006 ; Dashti et al. 2010). The centrifuge results here presented confirmed that observation and evidenced a dependence of the superficial settlement over $\mathrm{I}_{\mathrm{A}}$.

The occurrence of a significant share of co-seismic settlement during the first stages of the applied input motion (Figs. 5, 6 and 7) indicates a significant water discharge from the base upwards even when the excess pore pressure generation process still dominates on the dissipation process. In all the tested models, the settlement rate was higher before the onset of liquefaction than after liquefaction triggering or at the end of liquefaction, when only dissipation occurred. The higher settlement rate during pore pressure build-up than during the following stages can be interpreted as a temporary increase of soil hydraulic conductivity $\mathrm{k}$ which, in combination to the formation of hydraulic gradients, enhances the potential for pore water migration (Arulanandan and Sybico 1992; Su et al. 2009; Shahir et al. 2012).

A back analysis of the results shown in Figs. 5, 6 and 7 has been attempted following the method proposed by Su et al. 2009, with the aim of estimating the variation of k during the seismic loading. Assuming incompressibility of pore fluid and soil grains, the settlement rate at the soil surface has been assumed equal to the flow rate of water out of the soil surface. The hydraulic gradient has been determined assuming a linear distribution of excess pore pressure from the soil surface, where the excess pore pressure is zero, down to the depth of the shallower ppt (ppt4 in M1_GM31 and ppt5 in tests M1_GM17 and 34), as:

$$
i=\frac{\Delta u}{\left(\gamma_{f} z\right)}
$$

where $\gamma_{\mathrm{f}}$ is the unit weight of the pore fluid.

According to Darcy law, the flow rate plotted against the hydraulic gradient, gives an indication of $\mathrm{k}$ during the stages of pore pressure build-up, max $\mathrm{Ru}$ and subsequent reconsolidation. This is shown in Fig. 10. The settlement rate, rises very quickly to its maximum value during the first few seconds of dynamic excitation, i.e. as the pore pressure and the hydraulic gradient reach their maximum, according to an almost linear relationship. In this stage the hydraulic conductivity is several time larger than the pre-shock value of $2 \mathrm{~mm} / \mathrm{s}$. Then, in models GM17 and GM34, as the excess pore pressure start to decay, k decreases down a value slightly lower than the initial static value. In model GM31, while the soil experiences liquefaction (hydraulic gradient equal to the critical value, $\mathrm{i} \approx 1$ ), $\mathrm{k}$ rapidly decreases to the pre-shock value and then remains constant as dissipation starts and i reduces.

According to Shahir et al. (2012), the variation of hydraulic conductivity k during earthquake loading and consequent liquefaction can be expressed as a function of $\mathrm{Ru}$, adopting the following equation:

$$
\frac{k}{k_{i}}=\left\{\begin{array}{lc}
1+(\alpha-1) \cdot R_{u}^{\beta_{1}} & \text { for } R_{u}<1 \\
\alpha & \text { for } t_{1} \leq t<t_{2} \\
1+(\alpha-1) \cdot\left(\frac{t_{3}-t}{t_{3}-t_{2}}\right)^{\beta_{3}} & \text { for } t_{2} \leq t<t_{3} \\
1 \quad & \text { for } t_{3} \leq t
\end{array}\right.
$$

where $\mathrm{k}_{\mathrm{i}}$ is initial permeability coefficient before shaking, $\alpha, \beta_{1}, \beta_{3}$ are positive material constants, $t$ represents the current time, $t_{1}$ is the time of onset of initial liquefaction, time 


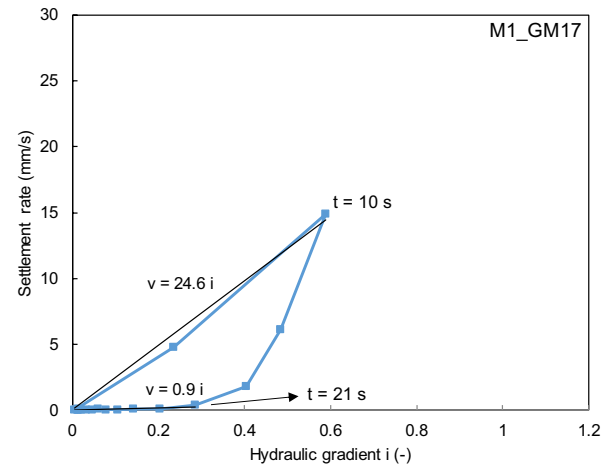

(a)

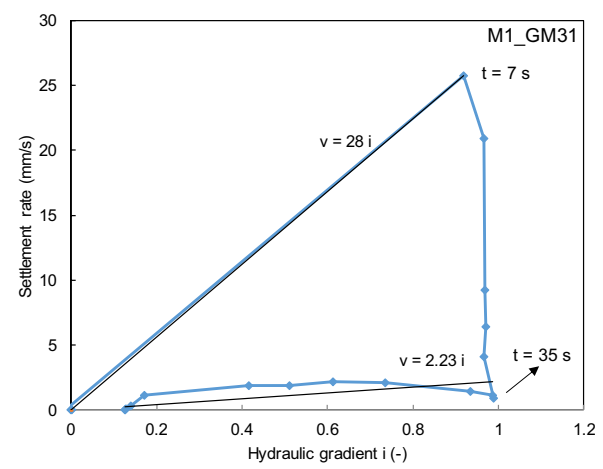

(c)

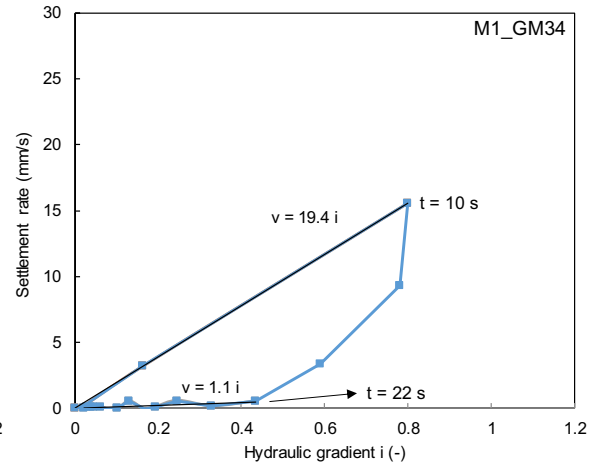

(b)

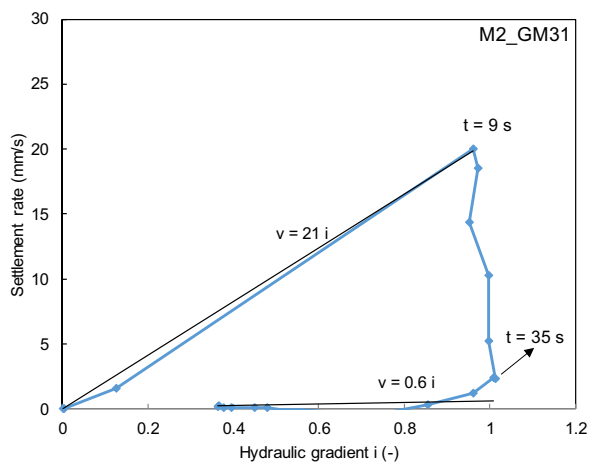

(d)

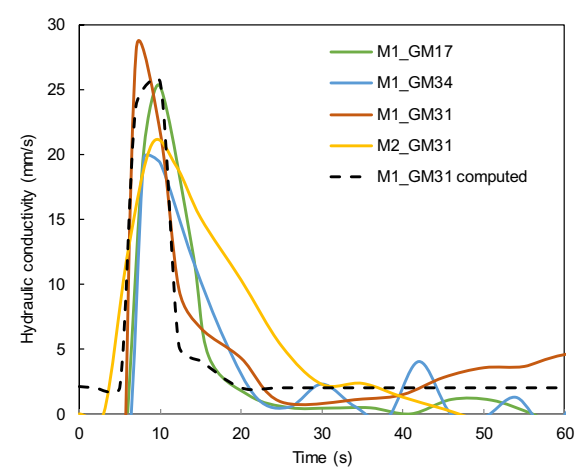

(e)

Fig.10 a M1_GM17, b M1_GM34, c M1_GM31, d M2_GM31: settlement rate vs hydraulic gradient. e hydraulic conductivity time history. Prototype scale

$t_{2}$ is used to determine the duration of peak permeability, during liquefaction state, time $t_{3}$ is a constant which specifies the end of $k$ variation process. The parameter $\alpha$ represents the maximum permeability variation respect to the initial value when the soil liquefies; $\beta_{1}$ defines the rate of permeability variation during the pore pressure increase up to liquefaction, $\beta_{3}=$ defines the rate of permeability reduction during dissipation phase. 


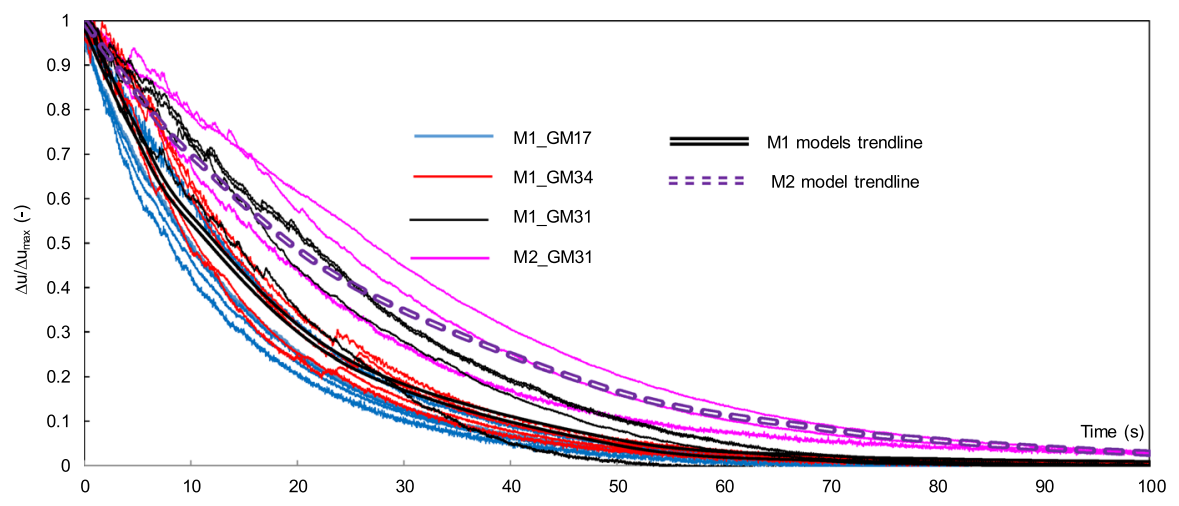

Fig. 11 Excess pore pressure decay of model M1_GM17, M1_GM34, M1_GM31, M2_GM31. Prototype scale

The results of model M1_GM31 shown in Figs. 7 and 10 have been back analysed to calibrate the material constant $\alpha, \beta_{1}, \beta_{3}, \mathrm{t}_{2}$ and $\mathrm{t}_{3}$, which resulted $\alpha=12.8, \beta_{1}=0.93, \beta_{3}=2$, $\mathrm{t}_{2}=13 \mathrm{~s}$, and $\mathrm{t}_{3}=20 \mathrm{~s}$, with $\mathrm{t}_{1}$ assumed equal to $8.5 \mathrm{~s}$. The so calibrated permeability function is shown in Fig. 10e. Accounting for a variable permeability function may be particularly useful when calibrating numerical models on centrifuge test results, as previous studies have shown that the adoption of constant permeability generally implies a significant underestimation of liquefaction induced settlement.

The dissipation of $\Delta \mathrm{u}$, once drainage became the dominant process during or at the end of the applied input motion, is shown in Fig. 11. The dissipation curves are normalised over the maximum $\Delta \mathrm{u}$ and the time scale of each curve is set to zero at the stating time of dissipation.

The dissipation curves of homogeneous models outline a unique family, with the dissipation being slightly faster in test GM17, slightly slower in test GM31.

A negative exponential function (Wang et al. 2013) can be adopted to interpret the whole family of dissipation curves:

$$
\frac{\Delta u}{\Delta u_{\max }}=e^{-\frac{t}{T_{d}}}
$$

where $T_{d}=d^{2} / c_{v}$ is a drainage period, function of the sand consolidation coefficient and of the drainage distance for excess pore pressure flows to the surface. $T_{d}$ resulted equal to $17 \mathrm{~s}$ and was computed assuming (i) a drainage distance equal to half height of the models; (ii) a confined modulus $\mathrm{M}=15 \mathrm{MPa}$, derived in the stress range $50-100 \mathrm{kPa}$ from an oedometric test on TS reconstituted at the void ratio of the centrifuge models; (iii) a pore fluid unit weight $\gamma_{\mathrm{f}}=9.84 \mathrm{kN} / \mathrm{m}^{3}$. To obtain the best fit of the experimental curves, the hydraulic conductivity assumed was $\mathrm{k}=0.0017 \mathrm{~m} / \mathrm{s}$, slightly lower than the pre-shock value and in accordance with the change in permeability experienced by the sand during the dynamic excitation.

\subsection{Stratified model M2, effect of a fine cap layer at the sandy deposit surface}

The stratified model M2_GM31 (Fig. 2c) was tested to investigate the effect of a top fine grained crust of lower permeability than the underlain sand on the liquefaction triggering, 
on liquefaction manifestations on the soil surface and on the post-earthquake behaviour. Fine grained layers typically cap liquefiable riverbed or floodplain sandy deposits, as does happen in the reference site of this experimentation, where liquefaction occurred during the Emilia Romagna 2012 seismic sequence. In flat areas of the reference prototype, the surface silty and clayey layer, about $2 \mathrm{~m}$ thick, which cover the liquefied riverbed sandy layer, was crossed by boilingpaths; in gently sloping areas the top layer was crossed by extensional fissure and slipped downward, indicating water film formation (Kokusho 1999, 2003; Kokusho and Fujita 2001) and lateral spreading. Damages were induced to existing building in both cases. In flat areas, buildings provided of water wells were not or were only marginally damaged, since wells acted as preferential dissipation paths of excess pore pressure, confirming the usefulness of drains as liquefaction mitigation technique.

In flat areas, the presence of a unliquefiable layer above a liquefiable sandy deposit may influence the manifestation of liquefaction depending on its thickness (Ishihara 1985). If the top layer is thin, the pore pressure from below may break the cap, generating ground ruptures. If the layer is sufficiently thick, its weight is enough to sustain the uplift force due to excess pore pressure from below and the effects of liquefaction at greater depths are not evident at the ground surface. The uplift pressure increases as the thickness of liquefiable layer rises. According to Ishihara 1985 and to observation of centrifuge tests by Fiegel and Kutter (1994), Brennan and Madabhushi (2005), in the layered model here presented, superficial manifestations of liquefaction were expected, being the top fine layer relatively thin respect to the thickness of the underlining liquefiable sand.

As to the input motion of the layered model, the shaking table fired a GM31 slightly stronger than the homogeneous GM31 model, both in terms of PGA (from $0.198 \mathrm{~g}$ to $0.243 \mathrm{~g}$ ) and intensity (from 0.601 to 0.673 ). On the other hand, in the first few seconds of the GMs, the energy release was slightly faster in M1 than in M2 as shown in Fig. 9b (brown and yellow lines).

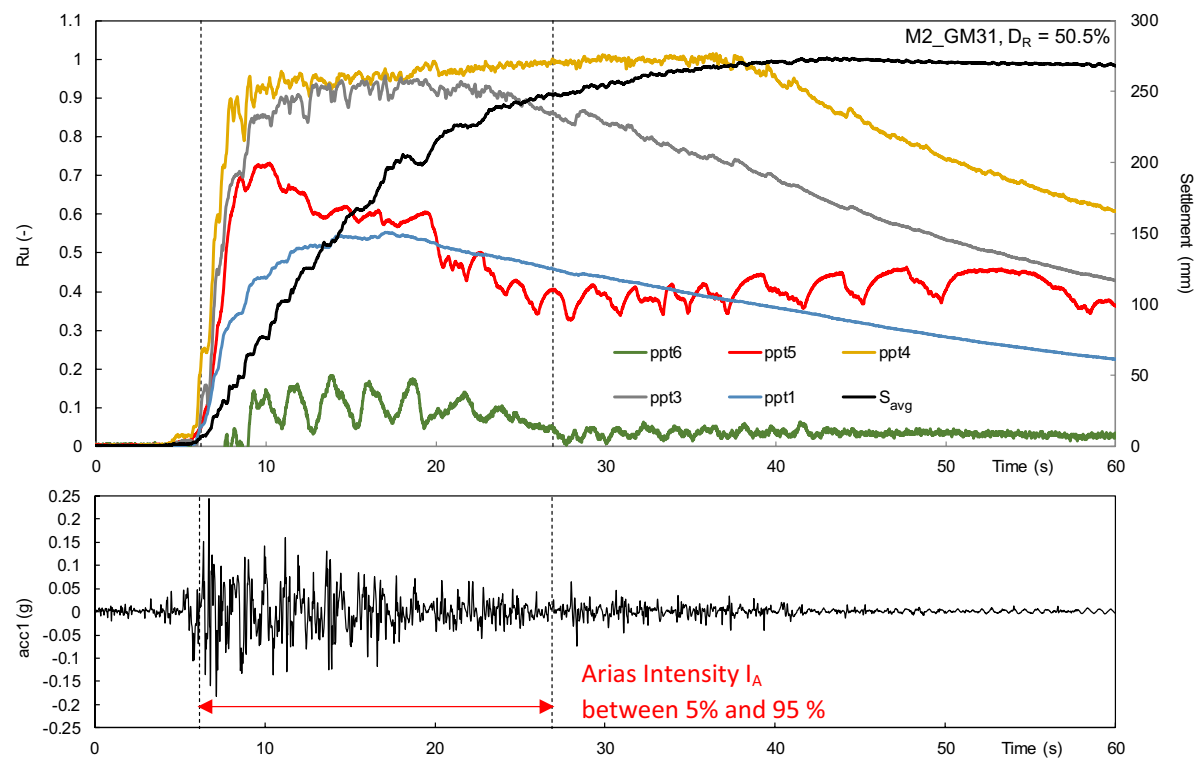

Fig. 12 Pore pressure ratio $\mathrm{Ru}$, average settlement $\mathrm{S}_{\mathrm{avg}}$ and input time history of model M2_GM31. Prototype scale 
The spectral ratios SR between acc6 and acc1 and the pore pressure ratios Ru registered during the shock, are displayed in Figs. 8 and 12, respectively.

It's worth noting that ppt6 was embedded in the fine layer: at the beginning of the inflight consolidation it registered lower pore pressure than the hydrostatic as an effect of the high initial overconsolidation ratio which generated matrix suction.

While liquefaction was reached at the depths of ppts 3 and 4, as indicated by the Ru time histories in Fig. 12 and by the de-amplification measured by acc6 (pimpled grey line in Fig. 8), at the depth of ppt5, Ru raised to a maximum value of 0.7 , then started to decay in advance respect to the end of the ground shaking, reached an almost constant value of 0.4 which was maintained for about $30 \mathrm{~s}$ after the end of the ground shaking. The Ru time history is characterised by continuous dilation induced suction spikes.

In Fig. 13 the isochrones of excess pore pressure of models M1_GM31 and M2_ GM31 are compared for instants ranging from 0.3 to 5 times the duration of the input earthquake. This figure shows that at depths greater than ppt5 location, model M2 experienced a co-seismic behaviour very similar to that observed for model M1, with the sand layer subdivided in a bottom part which didn't experienced liquefaction and the upper part which liquefied, as a combined effect of the seismic cyclic strain and the water flow from below. On the other hand, in the area where ppt5 was located, Ru significantly lower than 1 were measured. This behaviour can be justified by the presence of the fine layer which was consolidated in a one-dimensional consolidometer from a slurry, then, before the test, it was unloaded, trimmed and placed above the saturated sand model; during this process and before centrifuge spin-up, the layer experienced superficial desaturation phenomena which rose matrix suction. The layer may have induced a local desaturation of the sand surface immediately below the interface, where ppt5 was located, so that ppt5 experienced pre-shock pore pressure value smaller than hydrostatic equilibrium. As a consequence, the excess pore pressure induced by the dynamic loading and by the water flow from below, never reached $\mathrm{Ru}=1$.

However, the hydraulic gradient computed between ppts 5 and 6 in Fig. 13b, was similar to the critical value.

The presence of the low permeability fine crust, created a zone of accumulation of pore fluid, as indicated by constant values of Ru measured by ppt5 for about $30 \mathrm{~s}$ after

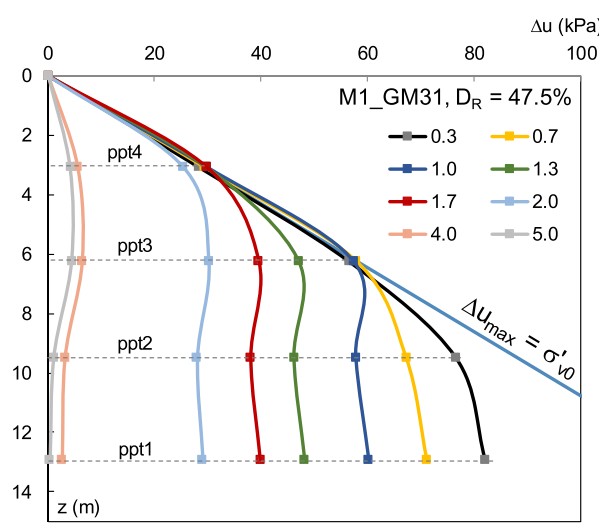

(a)

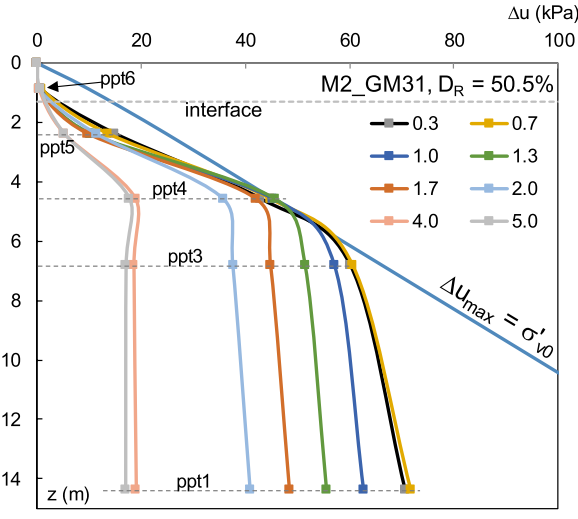

(b)

Fig. 13 Models a M1_GM31 and b M2_GM31. Isochrones of excess pore pressure. Prototype scale 


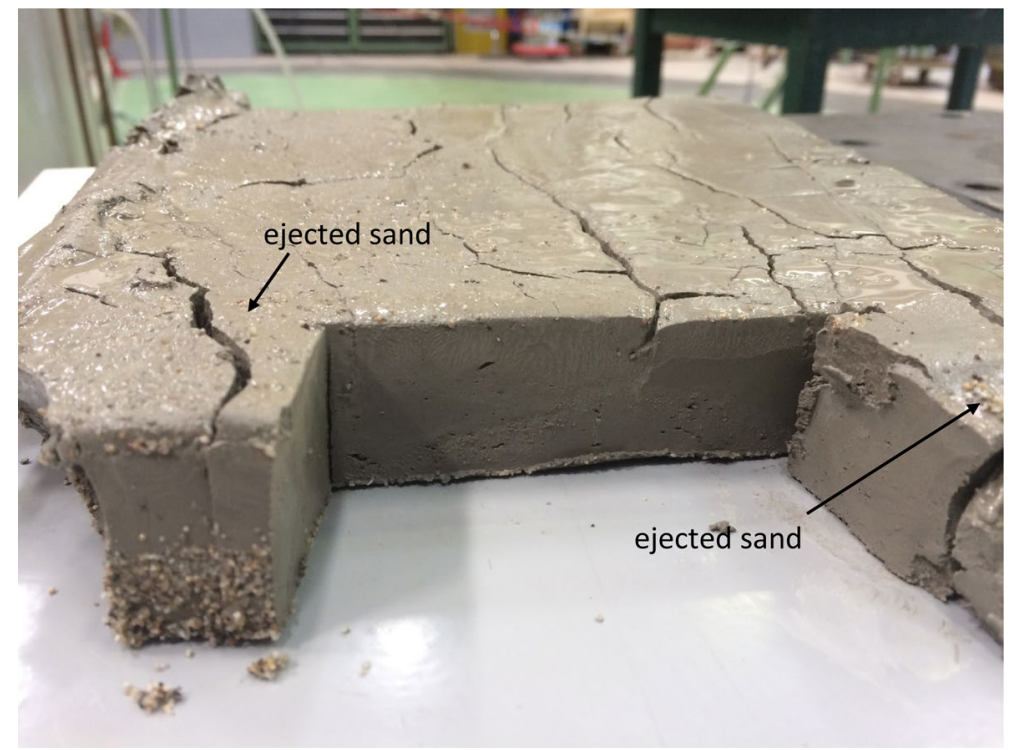

Fig. 14 M2_GM31: fine cap layer crossed by cracks and sand at the end of the test

the end of the ground motion. The pore fluid reached a pore pressure value sufficiently high to lift the fine cap (ppt5 measured $\Delta \mathrm{u}=37 \mathrm{kPa}$, the total overburden stress at the sand/cap interface being $27 \mathrm{kPa}$ ). Local bulging induced tension stresses which caused the formation of cracks and local high permeability channels and vents through which the fluid flow upward. At the end of the test the fine cap was diffusely crossed by transversal cracks and covered by fluid and a small amount of sand, as shown in the picture reported in Fig. 14.

The $\Delta \mathrm{u}$ build-up rate measured in M2_GM31 by ppts 3 and 4 was similar to that measured in the homogeneous ones, suggesting that the $\Delta \mathrm{u}$ generation depends on the seismic intensity and on state of the sand, and the presence of the cap has minor influence.

As evidenced in Figs. 11, 12 and 13, the dissipation rate was lower in presence of the fine cap. While in M1_GM31 model the excess pore pressure was almost entirely

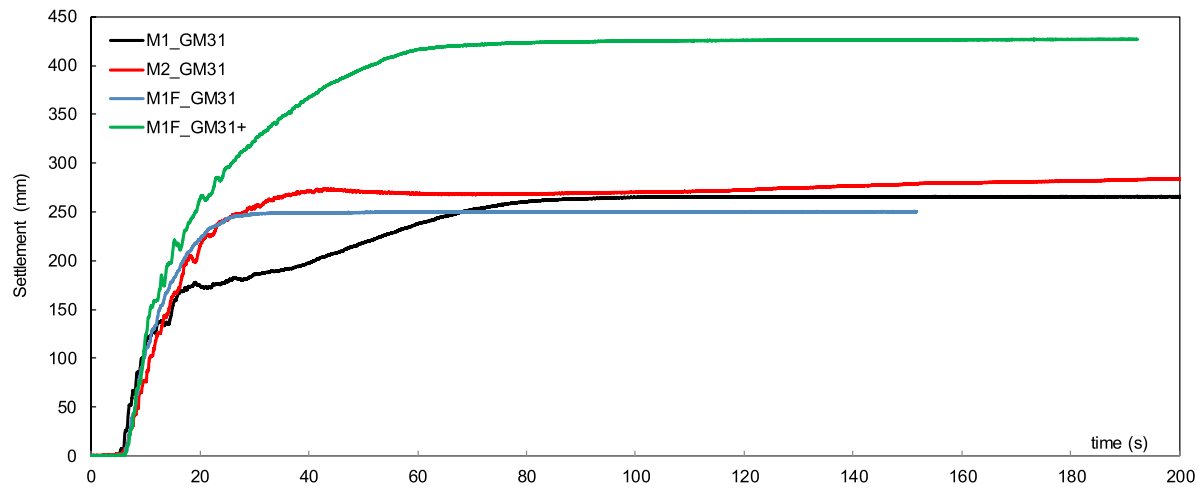

Fig. 15 GM31 and GM31 + models: free field average settlement $S_{\text {avg }}$ 
dissipated at all depths after about $70 \mathrm{~s}$ from the end of the earthquake, in the capped model M2_GM31 at $100 \mathrm{~s}$ (Fig. 11) $\Delta \mathrm{u}$ was at all depths still decreasing, indicating that the dissipation process was in progress, as an effect of the low permeability boundary at the top. Indeed, while the settlement rate of M1 zeroed after $70 \mathrm{~s}$ from the end of the earthquake, in model M2_S1 the settlement rate first zeroed, then restarted to increase and was equal to $0.23 \mathrm{~mm} / \mathrm{s}$ at the end of recording (Fig. 15). At the end of recording the average surface settlement was 265 mm in M1_GM31 and 284 mm in M2_GM31.

In Fig. 11, the average decay of $\Delta \mathrm{u} / \Delta \mathrm{u}_{\max }$ of model M2 has been fitted by a different trend line than models M1, characterised by a longer drainage period $T_{d}=28 \mathrm{~s}$, as an effect of the different boundary conditions. The best fit of the experimental curves has been obtained assuming the same confined modulus $\mathrm{M}$, pore fluid $\gamma_{\mathrm{f}}$ and hydraulic conductivity $\mathrm{k}$ as in the homogeneous model and a $1.5 \mathrm{~m}$ longer drainage path to account for the presence of the fissured crust.

The slower dissipation rate is also reflected by the settlement rate during the first few seconds of dynamic excitation, when the high hydraulic gradient onset an upward water discharge. In the layered model a bit lower settlement rate was measured in this stage, as can be observed in Figs. 9a and 10e. The hydraulic conductivity function in Fig. 10e was computed as a function of the surface settlement rate and the hydraulic gradient between soil surface and ppt4 (neglecting the non-linear distribution of $\Delta \mathrm{u}$ below the fine layer). The slower variation of the $\mathrm{k}$ function in the layered model, both before and after the attainment of the peak value, can be interpreted as a consequence of the lower drainage capacity of the sand due to the presence of the fine cap.

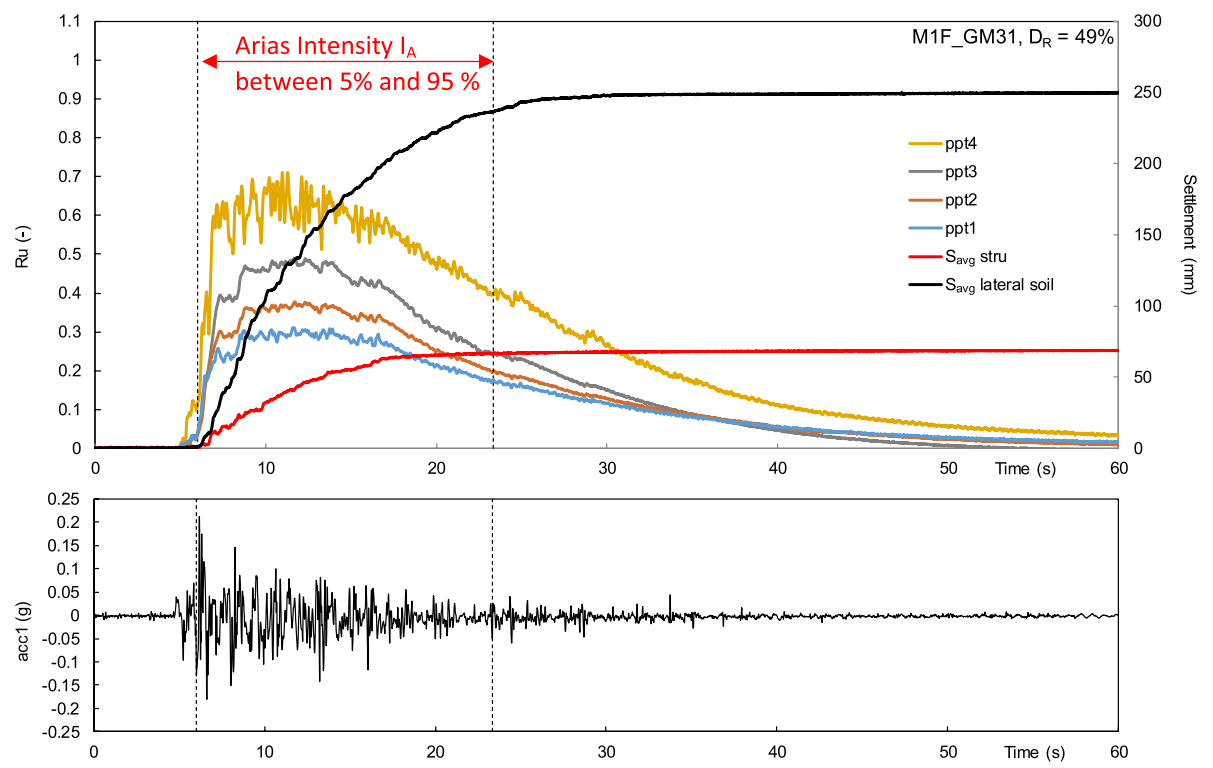

Fig. 16 Pore pressure ratio Ru, average settlement $S_{\text {avg }}$ of models M1F_GM31. Prototype scale 


\subsection{Presence of a model structure on shallow foundations, model M1F}

The effect of a structure on shallow foundations on the dynamic response of the sandy profile under study was investigated by model M1F (test layout in Fig. 2d, test result in Figs. 15 to 19). The model was subjected to two input motions: the one used in free field models GM31 which, respect to M1_GM31 had similar PGA $(0.216 \mathrm{~g}$ vs $0.198 \mathrm{~g})$ and duration (17.24 s vs $18.63 \mathrm{~s}$ ), but slightly lower $\mathrm{I}_{\mathrm{A}, \max }(0.481$ vs 0.601$)$; an amplified (+3 $\mathrm{dB})$ version of GM31, called GM31 +, characterized by longer duration $\left(\mathrm{d}_{90}=22.48 \mathrm{~s}\right)$, higher PGA 50\% (0.292 g), and higher Arias intensity (1.844 vs 0.601).

M1F was instrumented under the foundation. After the first shock, test M1F_GM31, the soil lateral to the structure developed as high final settlement $S_{t}$ as the free field model (250 mm vs $265 \mathrm{~mm}$, Fig. 15) indicating that the soil external to structure underwent similar volumetric strain and experienced liquefaction. In the co-seismic phase, the settlement $S_{f}$ of the lateral soil was even larger than that of the free field model, as evidenced in Fig. 15.

As to the instrumented area below the foundation, the excess pore pressure $\Delta \mathrm{u}$ recorded by ppt 4 was similar to the free field model but the soil did not reach liquefaction as evidence by Ru in Fig. 16 and by the amplification effects registered by acc4, SR in Fig. 18; this is due to the higher stress field induced by the model structure. Ru values were computed accounting for the increased stress field underneath the foundation as derived by the numerical simulation of the test (Özcebe et al. 2021).

Moreover, due to the larger confinement and higher soil density, the Ru profile of ppt 4 was characterized by several cycles, associable with the sand dilative response above the phase transformation line, behavior not observed in the free field model M1_GM31. At the depths of ppt1, ppt 2 and ppt3, the measured $\Delta u$ was significantly lower than in the free field model and the maximum $\mathrm{Ru}$ did not exceed 0.5 . In general, the pore pressure build-up was slower respect to the free field model subjected to a similar input motion. The lower

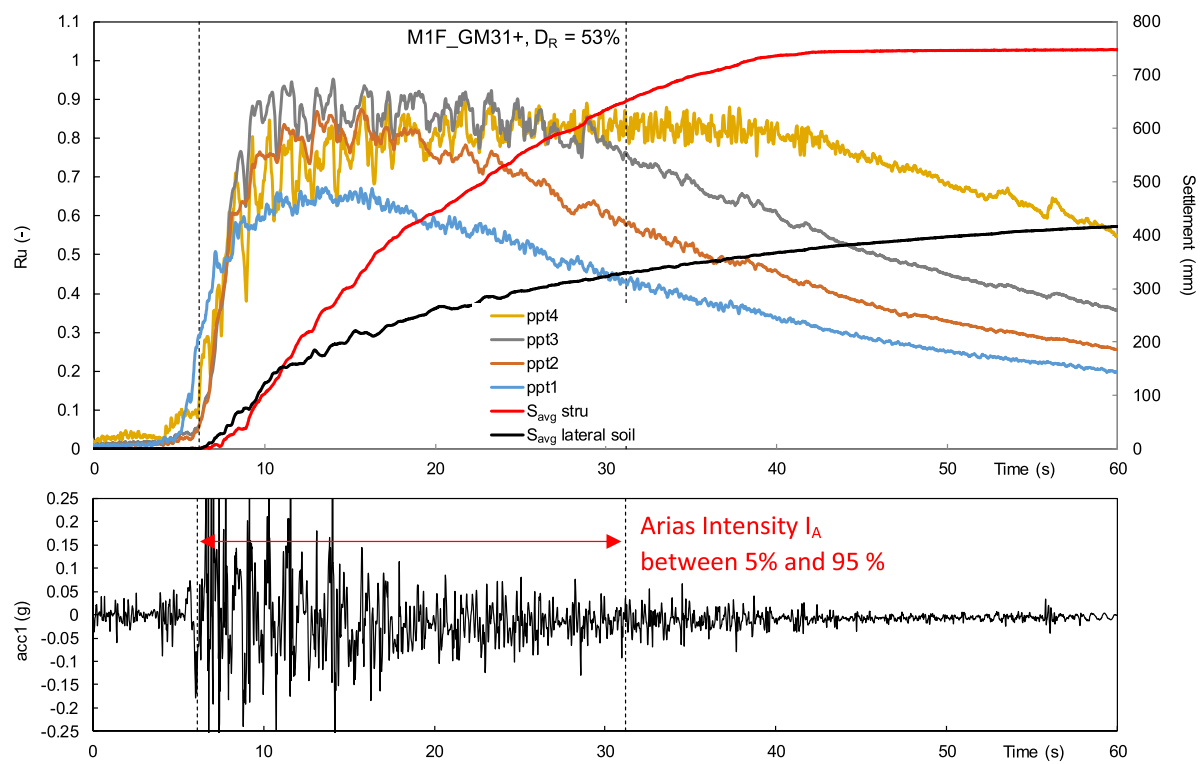

Fig. 17 Pore pressure ratio Ru, average settlement $S_{\text {avg }}$ of models M1F_GM31 +. Prototype scale 


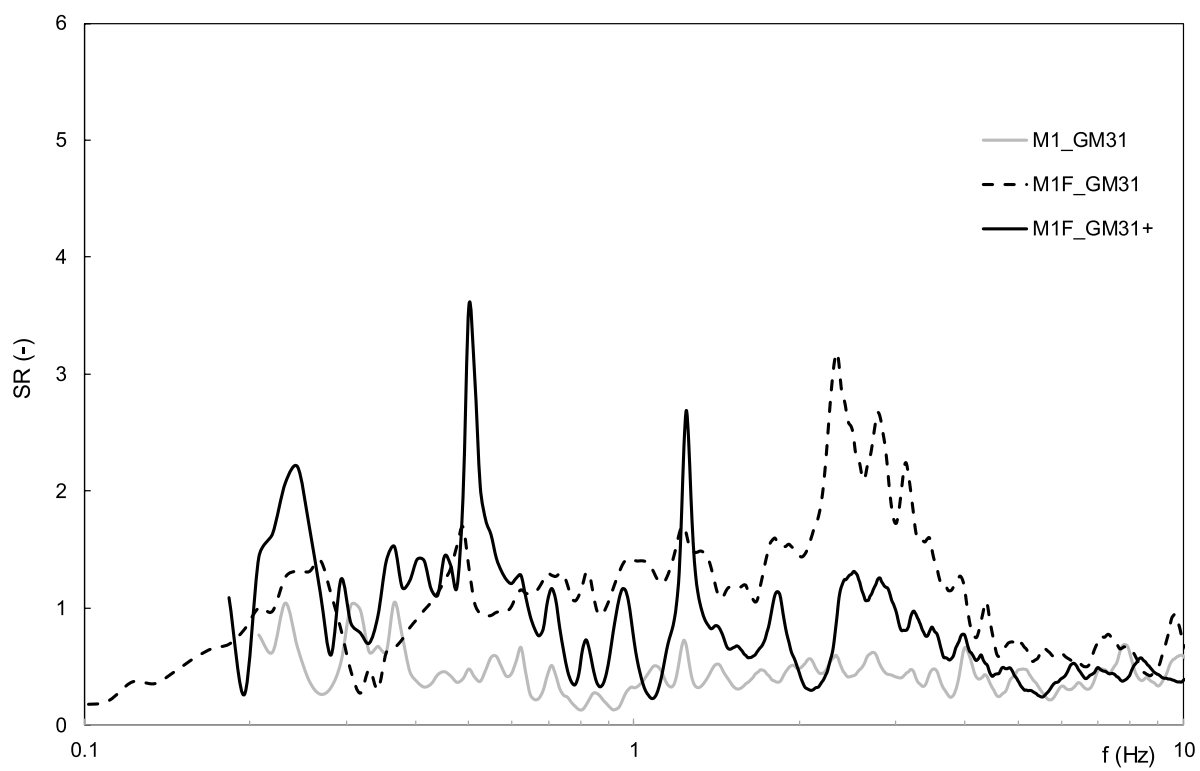

Fig. 18 Models M1_GM31, M1F_GM31 and M1F_GM31 + : spectral ratios computed dividing the FAS of the superficial accelerometer by the FAS of the base accelerometer. Prototype scale

$\Delta \mathrm{u}$ accumulation rate and the maximum $\mathrm{Ru}$ achieved under the structure can be explained as the effect of the larger confining stress and the deviatoric stress due to the foundation load which limited cyclic stress reversal and prevented liquefaction under the structure (Liu and Dobry 1997, Yang and Sze, 2011).

The excess pore pressure dissipation due to partial drainage during the ground shaking became at all depths the dominant process over $\Delta \mathrm{u}$ generation well in advance to the end of the earthquake, even if inward fluid flow may have occurred from the lateral free field which liquefied. The partial drainage triggered immediately after the first loading cycle, as evidenced by the settlements of both the free field and the structure, which began immediately to accumulate. At the end of recording the structure settled much less than the lateral free field (69 mm vs $250 \mathrm{~mm}$, Fig. 16); the structure settlement was entirely co-seismic and stopped before the end of the ground motion.

Both the free-field and structure settlement had similar accumulation function as the Arias Intensity, as shown in Fig. 19.

Overall, the effect of pore pressure accumulation due to the seismic induced shear strains and of water migration due to transient hydraulic gradient within the model was exceeded under the foundation by the positive effect of the confining and shear static stress which prevented full liquefaction to be achieved.

To induce liquefaction to the sand under the foundation and to observe the effect of liquefaction on the structure, the model was subjected to GM31+, which induced cyclic shear stresses sufficiently high to reduce and nullify the effect of the static shear load. It generated under the foundation similar $\Delta \mathrm{u}$ as those measured in the M1_GM31 free field model at all depths. 


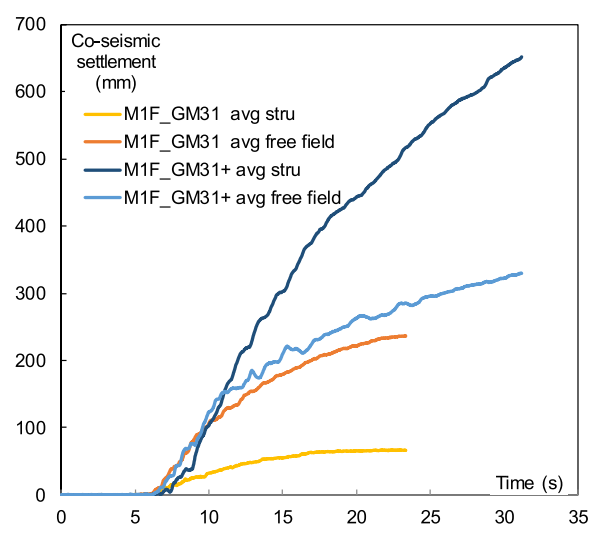

(a)

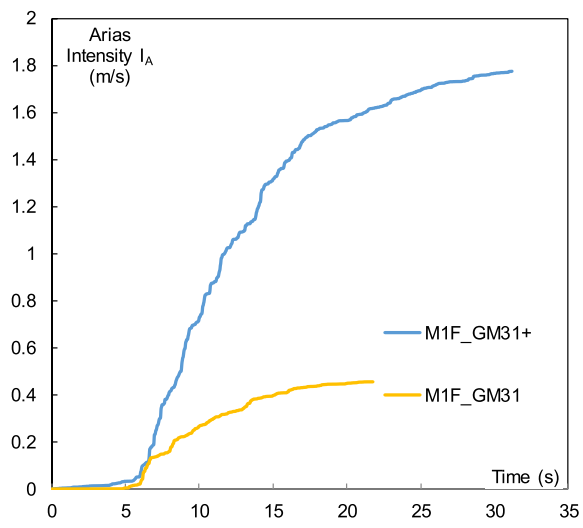

(b)

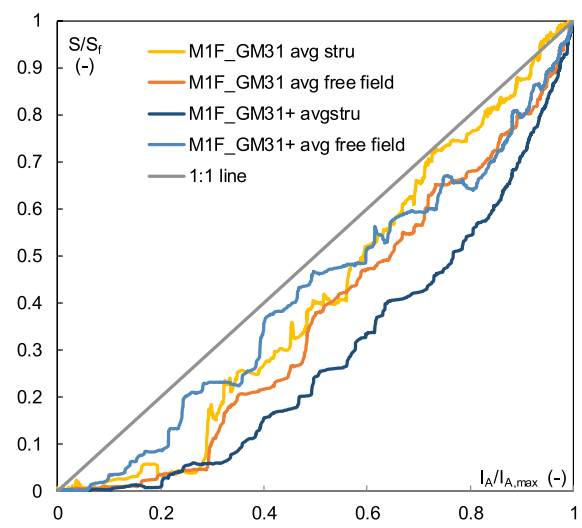

(c)

Fig. 19 Models M1F_GM31 and M1F_GM31 + : a co-seismic settlement b Arias Intensity time history and c normalized settlement vs normalized Arias Intensity. Prototype scale

The pore pressure ratio $\mathrm{Ru}$ at ppt4 (Fig. 17) quickly rose to 0.9 and remained constant all along the seismic excitation and several second thereafter, as a result of upward and possibly inward fluid flow from the bottom and from the free field of the model. At greater depths, ppts 2 and 3 also reached $R u=0.9$ but the liquefaction condition lasted less than the input motion. At the model base (ppt1) the maximum Ru not exceeded 0.65 and was attained for few seconds, whereupon the dissipation prevailed on cyclic induced excess pore pressure generation. Once the dynamic excitation finished, solidification of the sand layer was completed within $10 \mathrm{~s}$.

The spectral ratio in Fig. 18 confirms the occurrence of liquefaction, with de-amplification of almost all the frequencies higher than $0.8 \mathrm{~Hz}$.

The Ru profiles of ppts $2-4$ in Fig. 17 are characterised by more frequent and larger dilation cycles than under the less intense GM31, as a consequence of the slightly higher density attained by the foundation soil after the first shock.

A slower rate of pore pressure generation underneath the structure and smaller flow tendencies away from the foundations respect to the free field model can be observed in 
Fig. 17. However, the slower accumulation rate had negligible effect on the structure settlement, which onset immediately after the first loading cycles and, quickly got larger than that measured in the lateral soil after few seconds of excitation.

The structure experienced a total settlement of about $750 \mathrm{~mm}$ (Fig. 17), due to the combined effect of building-induced shear deformations and volumetric strains during partially drained cyclic loading, sedimentation and re-consolidation. The final building settlement was larger than the lateral free field settlement (about $430 \mathrm{~mm}$ ), consistently with observation of previous researchers, such as Liu and Dobry 1997, Dashti et al. 2010. About $90 \%$ of the structure settlement was co-seismic, the remaining $10 \%$ developed in $10 \mathrm{~s}$ after the end of the shock.

Overall the structure experienced a punching type deformation, with limited tilting $\left(1.3^{\circ}\right)$. The larger settlement than the lateral free field was caused by the adverse effects of concentrated shear strains near the foundation level, due to the vibration of structure, which caused progressive shear failures underneath the left and right foundations (Özcebe et al. 2021).

As to the lateral soil, the co-seismic share due to pore fluid drainage and discharge, sedimentation and consolidation was $78 \%$ of the final settlement. The final free field settlement under GM31 + was much higher than that of the model M1_GM31 (430 mm vs $265 \mathrm{~mm}$ ) indicating that the stronger earthquake induced liquefaction in a larger volume of sand than GM31. This may also explain the slower $\Delta \mathrm{u}$ dissipation rate under the foundation, which, while dissipating upward the accumulated $\Delta \mathrm{u}$, was probably interested by inflow from the lateral soil.

\section{Remarks}

The paper illustrates some results of a campaign of centrifuge tests carried out to study the liquefaction triggering mechanisms in sandy models, and to understand the effect of the following variables on co-seismic and post-seismic behaviours:

1. input motion,

2. fine crust at the surface

3. presence of a structure on shallow foundation

The tests have shown that the seismic behaviour of a saturated sandy deposit is a complex phenomenon during which several mechanisms coexist. The vibrated sand tends to contract and generate pore pressure. The generation of pore pressure produces transient hydraulic gradients and triggers pore fluid flow towards the drainage boundaries. The effect of fluid flow adds up to the effect of cyclic volumetric strain and seismic excess pore pressure can be enhanced by fluid flow induced $\Delta \mathrm{u}$. As a consequence, the measured $\Delta \mathrm{u}$ is a balance between generation, dissipation and inflow. The development of surface settlement, which quickly increases during the first loading cycles, confirms the importance of partial drainage during seismic excitation and, in general, the settlement due to rapid drainage during the initial earthquake stage of high hydraulic gradient development, can be greater than post-earthquake reconsolidation settlement, as in the tests here presented. When liquefaction occurs, also the sedimentation settlement can represent a significant share of the final superficial settlement. 
In the tests presented in this paper, the settlement rate resulted higher at the beginning of the applied earthquakes than in the post-consolidation phase, and this behavior can be interpreted as a temporary increase of soil hydraulic conductivity $\mathrm{k}$. The back analysis of the centrifuge tests have allowed to estimate the function of variability of a $\mathrm{k}$ as a function of the surface settlement rate. The hydraulic conductivity rises very quickly in the first few second of cyclic loading, then as much quickly falls down to slightly lower value than the pre-shock value, indicating that is during the very first seconds of seismic excitation that the high hydraulic transient gradients and partial drainage enhance the soil particle dispersion and the potential for volumetric deformations.

The function of surface settlement increase resulted in the presented test well correlated to the Arias Intensity function, and an almost linear dependency between the two normalized variables has been found, suggesting the possibility of using alternative earthquake parameter than PGA, such as an intensity measure, to evaluate the consequence of liquefaction.

The tests have evidenced that the presence of a top unliquefiable layer above the liquefiable sand, mainly influences the dissipation rate of the accumulated excess pore pressure. The top layer tested in the present experimentation was too thin to avoid superficial manifestation of liquefaction. The weight of the layer was lower than the pore pressure at the interface and the layer was lifted, bulged and cracked, with the formation of preferential permeability channels through which the fluid flow outside.

The $\Delta \mathrm{u}$ build-up rate resulted similar in presence or in absence of the cap layer, suggesting that the $\Delta \mathrm{u}$ generation depends on the seismic intensity and on state of the sand, and the presence of the cap has minor influence. On the other hand, dissipation took place slower and the development of post-earthquake consolidation settlement lasted longer.

The presence of a superficial structure on shallow foundations evidenced two opposite mechanisms. The higher confinement stress field induced by the static load and the presence of static shear stress is beneficial when the earthquake is not strong enough to induce liquefaction under the foundation and overcomes the effect of pore pressure accumulation due to the seismic induced shear strains and water migration.

If the underlain soil liquefies, the combined effect of building-induced shear deformations and volumetric strains during partially drained cyclic loading, sedimentation and reconsolidation can induce building sinking.

Acknowledgements This research has been carried out within the framework of the European LIQUEFACT project. The LIQUEFACT project has received funding from the European Union's Horizon 2020 Re-search and Innovation Programme under Grant Agreement No. 700748. This support is gratefully acknowledged by the authors.

Funding Open access funding provided by Università degli studi di Bergamo within the CRUI-CARE Agreement.

Open Access This article is licensed under a Creative Commons Attribution 4.0 International License, which permits use, sharing, adaptation, distribution and reproduction in any medium or format, as long as you give appropriate credit to the original author(s) and the source, provide a link to the Creative Commons licence, and indicate if changes were made. The images or other third party material in this article are included in the article's Creative Commons licence, unless indicated otherwise in a credit line to the material. If material is not included in the article's Creative Commons licence and your intended use is not permitted by statutory regulation or exceeds the permitted use, you will need to obtain permission directly from the copyright holder. To view a copy of this licence, visit http://creativecommons.org/licenses/by/4.0/. 


\section{References}

Adamidis O, Madabhushi SPG (2018) Experimental investigation of drainage during earthquake-induced liquefaction. Géotechnique 68(8):655-665. https://doi.org/10.1680/jgeot.16.P.090

Arulanandan K, Sybico J (1992) Post-liquefaction settlement of sand. Proceeding of the wroth memorial symposium, England, Oxford University, pp 94-110

Airoldi S, Fioravante V, Giretti D (2016) Soil liquefaction tests in the ISMGEO geotechnical centrifuge. Proceedings of China-Europe conference on geotechnical engineering, pp 469-472

Airoldi S, Fioravante V, Giretti D, Moglie J (2018) Report on validation of retrofitting techniques from small scale models. Deliverable D4.2 of Liquefact project, funded under the European Union's Horizon 2020 Research and Innovation Programme, GA No. 700748

Baldi G, Belloni G, Maggioni W (1988) The ISMES Geotechnical Centrifuge. In: Corte' JF (ed) Centrifuge 88, Paris. Balkema, Rotterdam, pp 45-48.

Brennan AJ, Madabhushi SPG (2005) Liquefaction and drainage in stratified soil. J Geotech Geoenviron Eng 31(7):876-885

Brennan, A. J., Madabhushi, S. P. G., and Houghton, N. E. (2006). Comparing laminar and equivalent shear beam (ESB) containers for dynamic centrifuge modelling. Proc. 6th Int. conf. on physical modelling in geotechnics 06, Vol. 1, Balkema, Rotterdam, The Nertherlands, pp 171-176.

Calabrese L, Martelli L, Severi P (2012) Stratigrafia dell'area interessata dai fenomeni di liquefazione durante il terremoto dell'Emilia (Maggio 2012). In: Proceedings of the 31st GNGTS, november 20-22, Potenza, pp 119-125.

Chiaradonna A, Özcebe AG, Bozzoni F, Fama A, Zuccolo E, Lai CG, Flora A, Cosentini RM, d'Onofrio A, Bilotta E, Silvestri F (2018). Numerical simulation of soil liquefaction during the 20 May 2012 M6.1 Emilia Earthquake in Northern Italy: the case study of Pieve di Cento. In Proceedings of the 16th European conference on earthquake engineering, 16ECEE, Thessaloniki, Grecia, 18-21 June 2018. Paper 11234.

Dashti S, Bray JD, Pestana JM, Riemer M, Wilson D (2010) Mechanism of seismically induced settlement of buildings with shallow foundation on liquefiable soil. J Geotech Geoenviron Engng ASCE 136(1):151-164

Fasano G, De Sarno D, Bilotta E, Flora A (2019) Design of horizontal drains for the mitigation of liquefaction risk. Soil Founda 59:1537-1551

Fiegel GL, Kutter B (1994) Liquefaction mechanism for layered soils. J Geotech Eng 120(4):737-755

Fioravante V, Giretti D (2016) Unidirectional cyclic resistance of Ticino and Toyoura sands from centrifuge cone penetration tests. Acta Geotech 11(4):953-968. https://doi.org/10.1007/s11440-015-0419-3

Fioravante, V. and Jamiolkowski, M (2005). Physical modelling of piled rafts. Soil structure interaction: Calculation methods and Engineering Practice. International geotechnical conference. Saint Petersburg. May 2005, 89-95.

Giretti D, Fioravante V (2017) A correlation to evaluate cyclic resistance from CPT applied to a case history. Bull Earthquake Eng. https://doi.org/10.1007/s10518-016-0057-7

Green RA, Mitchell JK (2003) A closer look at Arias intensity-based liquefaction evaluation procedures. Proceedings of $7^{\text {th }}$ Pacific conference on earthquake engineering, 13-15 February, paper no. 94. Christchurch, New Zealand: University of Canterbury, p 9

Ishihara (1985). Stability of natural deposits during earthquakes. Proceedings of the international conference on soil mechanics and foundation engineering. Balkema, $\mathrm{R}$ otterdam, pp 321-376

Kayen RE, Mitchell JK (1997) Assessment of liquefaction potential during earthquakes by Arias intensity. J Geotech Geoenviron Eng 123(12):1162-1174

Kokusho T (1999) Water film in liquefied sand and its effect on lateral spread. J Geotech Geoenviron Eng 125(10):817-825

Kokusho T (2003) Current state of research on flow failure considering void redistribution in liquefied deposits. Soil Dyn Earthq Eng 23:585-603

Kokusho T, Fujita K. (2001). Water films involved in post-liquefaction flow failure in Niigata City during the 1964 Niigata earthquake. Proceedings of the recent advances in geotechnical earthquake engineering and soil dynamics, San Diego, CD publication

Kramer S, Mitchell JK (2006) Ground Motion Intensity Measures for Liquefaction Hazard Evaluation. Earthq Spectra 22(2):413-438. https://doi.org/10.1193/1.219497

Kutter, B.L. (1995). Recent Advances in Centrifuge Modeling of Seismic Shaking. Proceedings of the 3rd international conference on recent advances in geotechnical earthquake engineering and soil dynamics, April 2-7, 1995, Volume II, St. Louis, Missouri, 927-941

Li X-S, Wang Z-L (1998) Linear representation of steady state line for sand. J Geotech Geoenviron Eng ASCE 124(12):1215-1217 
Liu L, Dobry R (1997) Seismic response of shallow foundation on liquefiable sand. J Geotech Geoenviron Engng ASCE 123(6):557-567

Özcebe AG, Giretti D, Bozzoni F, Fioravante V, Lai CG (2021) Centrifuge and numerical modelling of earthquake-induced soil liquefaction under free-field conditions and by considering soil-structure interaction. Bull Earthq Eng 19(1):47-75. https://doi.org/10.1007/s10518-020-00972-3

Scott RF (1986) Solidification and consolidation of a liquefied sand column. Soils Founda 26(4):23-31

Shahir H, Pak A, Taiebat M, Jeremic B (2012) Evaluation of variation of permeability in liquefiable soil under earthquake loading. Comput Geotech 40:74-88

Su D, Li X-S, Xing F (2009) Estimation of the apparent permeability in the dynamic centrifuge tests. Geotech Test J 32(2):22-30

Yang J, Sze HY (2011) Cyclic behaviour and resistance of saturated sand under non-symmetrical loading conditions. Géotechnique 61(1):59-73

Wang B, Zen K, Chen GQ, Zhang YB, Kasama K (2013) Excess pore pressure dissipation and solidification after liquefaction of saturated sand deposits. Soil Dyn Earthq Eng 49:157-164

Zeng X, Schofield AN (1996) Design and performance of an Equivalent Shear Beam (ESB) model container for earthquake centrifuge modelling. Geotechnique 46(1):83-100

Publisher's Note Springer Nature remains neutral with regard to jurisdictional claims in published maps and institutional affiliations. 